\title{
An adaptive optimization procedure for spot-welded structures
}

\author{
Q.I. Bhatti, M. Ouisse*, S. Cogan \\ FEMTO-ST Institute, Applied Mechanics - UMR CNRS 6174, 24, chemin de l'épitaphe, 25000 Besançon, France
}

\begin{abstract}
The behavior of a spot welded structure under dynamic loads is strongly influenced by the number and locations of the resistance spot welds. The design problem requires the number and locations of spot welds to be optimized so as to obtain reasonable trade-offs between manufacturing cost and structural performance. An adaptive optimization procedure is proposed which iteratively adds and removes spot welds based on decision indicators in order to correct for the approximations made in the iterative pro-cess. In practice, it is possible that some spot welds may be defective or even missing when structure leaves the assembly line. Therefore, a simple robustness indicator is formulated to characterize the impact of the number of defective or missing spot welds on the system performance. This indicator pro-vides a useful decision making tool for deciding both how many spot welds should be inspected following assembly as well as pointing to a small number of critical spot welds that should be reinforced. The pro-posed methodologies will be illustrated on a full body-in-white structure for a car.
\end{abstract}

\section{Introduction}

Resistance spot welding is one of the main manufacturing techniques for sheet metal structures and the automotive industry, for example, uses thousands of resistance spot welds (abbreviated RSW or spot weld) to assemble the body-in-white (BIW) for vehicles. Meanwhile, global competition pushes the automotive industry to reduce manufacturing cost and spot welds represent a significant contribution to the overall cost of a vehicle. Therefore, it is a worthwhile task to reduce the number of RSWs on the vehicle without compromising the performance. Due to mass production, even a small reduction in their number could lead to substantial reduction in the cost. However, the number of spot welds and their spatial distribution has a significant impact on the structural performance criteria that must be taken into account by an analyst, including the static, dynamic, and crash behaviors.

Currently, the number and locations of spot welds is largely based on the designer's know-how. However, this proves to be a daunting task for even the most experienced designers and problem has not been fully addressed by the research community. In the context of optimization of spot welded structures, the problem has been formulated in two ways: performance optimization and quantity optimization.

In Performance Optimization, total number of RSWs is fixed and defined a priori, based on the designer's know-how, experience and

\footnotetext{
* Corresponding author. Tel.: +33 381666046; fax: +33 381666700.

E-mail addresses: qamar.bhatti@femto-st.fr (Q.I. Bhatti), morvan.ouisse@univfcomte.fr (M. Ouisse).
}

an initial design. Here, the objective is to find the best spatial distribution of the fixed number of RSWs which improves the performance criteria of interest.

Some authors have examined the issue of improving the performance criteria by optimally relocating a fixed number of spot welds in the structure. In particular, Zhang and Taylor [1] proposed an optimization algorithm for a simple plate structure containing two spot welds allowing to modify the stiffness of the structure so as to maximize the fatigue life. They noted that the problem is strongly nonlinear even for only two spot welds. Similarly, Chae et al. [2] attempted to find the optimal locations of the spot welds in a plate structure containing three RSWs subjected to axial and transverse loads in order to maximize the load carrying ability of the structure. They also mentioned that the mesh should be refined and uniform in the vicinity of the RSWs for reliable results. Recently, Ertas and Sonmez [3] proposed another optimization algorithm for the same problem based on a Nelder-Mead Simplex method and stated that the results are comparable to those in [2].

However, attempting to solve the optimization problem based on a fixed number of spot welds, where one is interested in finding the optimal locations, can pose two problems. First, this number may be too small and the solution may not be feasible even for the best distribution. Secondly, a priori defined number of RSWs may be too large and the overall production cost will be high due to the presence of redundant spot welds in abundance. This suggests that not only the locations but also the number of RSWs should be included in the optimization process as a variable to be determined. This leads to the development of second formulation. 
In quantity optimization, the aim is to minimize the number of RSWs and find the optimal distribution of the existing number of RSWs simultaneously so as to ensure an acceptable level of performance.

Wang et al. [4] used this formulation at a full vehicle level and proposed a procedure to reduce the number of spot welds by maximizing the sum of the first torsional and bending eigenfrequencies of the structure considering each RSW as an independent design variable. They suggested that about $20 \%$ of the RSWs could be removed without any significant change in the behavior of the structure. Hasegawa et al. [5] optimized the locations and number of RSWs on a structure containing fifteen RSWs through a hybrid meta-heuristics approach where the optimal structure has spot welds of different nugget sizes. It is worthwhile noting that RSWs with different nugget diameters might be difficult to realize in real world. Similarly, Liao [6] used a genetic algorithm to find the optimal distribution of RSWs. Although meta-heuristic approaches are effective for this type of problem, they require a huge number of objective function evaluations which prohibits their use for large structures containing thousands of spot welds. Recently, Ouisse and Cogan [7] proposed an efficient procedure to remove redundant spot welds quickly.

Both types of optimization formulations discussed above can be handled by two strategies. Firstly, an over-populated spot welded structure is created and the least influential RSWs are removed from the structure in an iterative optimization procedure. In a second strategy, unnecessary spot welds are removed as before but in addition, spot welds are positioned in areas where their contribution is maximum either by moving the existing RSWs or by creating new spot welds.

Although numerical simulation time for large and complex structures has been reduced over the years, the iterative nature of the discrete optimization problem still requires careful attention to calculation costs. Hence, in order to optimize the number of spot welds in structures containing thousands of RSWs in a reasonable time, a simple decision making indicator is needed which can predict the contribution of individual RSW towards the performance criteria. This indicator will not only be helpful to find the locations of the most influential RSWs but will also serve to indicate the redundant RSWs whose contributions towards the performance criteria are negligible.

Bearing this in mind, we present in this paper a thorough study which was performed to investigate the correlation of different indicators with respect to the modal characteristics initially used by Ouisse and Cogan [7] in their optimization procedure to remove redundant spot welds. Furthermore, they used the first strategy to solve the optimization problem while in this paper, we propose an adaptive procedure based on the second strategy to solve the optimization problem. The optimization procedure uses the best indicator identified in this study to remove the redundant spot welds and simultaneously, adds the new spot welds in the proximity of the most influential RSWs.

Another aspect of this study concerns the impact of uncertainty in the form of missing or defective RSWs on the structural performance. Indeed, when a BIW leaves the assembly line it is not unusual to find a small percentage of spot welds missing. Moreover, fatigue effects through the lifetime of the vehicle can lead to the breakage of spot welds. The important question to address here is just how many RSWs can be defective without compromising the specified performance criteria.

In $[8,7]$, authors have used Monte Carlo (MC) simulations to study this problem under the assumption that each spot weld has equal chance of being defective or missing. However, a large number of analyses required for a meaningful MC simulation renders its use infeasible. Hence, we propose here a simple and less costly approach based on the impact of the most influential spot welds on the performance attribute of interest. The objective is to plot a robustness curve showing the evolution of performance when progressively more influential spot welds are defective or missing. Moreover, the robustness curve provides a useful decision making tool for deciding both how many spot welds should be inspected following assembly as well as pointing to a small number of critical spot welds that should be reinforced.

This presentation is organized as follows. In Section 2 the aspects of spot welds finite element (FE) modeling and the simplified FE representation used are briefly discussed. Then, the mathematical expressions of different indicators and their correlation with eigenfrequencies are presented in Section 3. Section 4 outlines the proposed adaptive optimization procedure and the results are discussed and compared with the non-adaptive method. In Section 5 an iterative procedure is proposed to obtain the robustness curves and followed by the description of a methodology to guarantee a specific level of robustness with the quality control of a small percentage of the most influential spot welds. At the end, the conclusions of the study are presented.

\section{Finite element modeling of the spot welds}

Although spot welding is an extensively used assembly technique for sheet metal parts, the presence of different physical and material properties in the proximity of the spot welds (e.g., geometrical irregularities, residual stresses and defects due to spot welding process, etc.) renders the process difficult to model accurately. However, various studies have been performed to represent the spot welds using a variety of local finite element models that realistically simulate their behavior under certain conditions. Indeed, several models can be found in the literature for different type of analysis, including: static, dynamic, crash, and fatigue behaviors. Palmonella et al. [9] presented a comprehensive review of different models for stress and stiffness prediction in dynamic structural analysis. Donders et al. [8] focused on the models to be used for large scale NVH (noise, vibration and harshness) and durability prediction in an industrial vehicle development environment. Moreover, thorough guidelines for the implementation of two of the models for structural dynamics are provided in Palmonella et al. [10].

Finite element models for RSWs can be divided into coarse and refined models. The former are simple models with few degrees of freedom (dofs) used for simulating global characteristics (e.g., stiffness, mass, etc.). The latter are more complex models to simulate smooth local physical effects (i.e., stress and strain in the proximity of a RSW.). For example, in the commercial finite element software MSC/NASTRAN: single beam, single brick, CWELD (special shear flexible beam type element), combination of elastic and rigid elements (e.g., CELAS, CBUSH.) belong to the coarse models class while solid bricks and umbrella models belong to the class of refined models. It is important to note that coarse and refined models are not always interchangeable, for example, a refined model used for accurately predicting stress may not be efficient to estimate the forces interchanged between the RSW and the structure or the stiffness [10].

The concept of spot weld optimization implies the ability to create new spot welds or remove existing ones when and where necessary at each step of the optimization process. However, creating new RSW may require a local remeshing of the structure depending on the type of RSW model used. Moreover, the FE spot weld models which can be conveniently and automatically added/removed at different locations in the structure are required for this process. In summary, while selecting a FE model of the RSW, it is important to consider the points such as simulation of global/local behavior (i.e., stress, strain, mass, force, etc.), FE modeling cost 


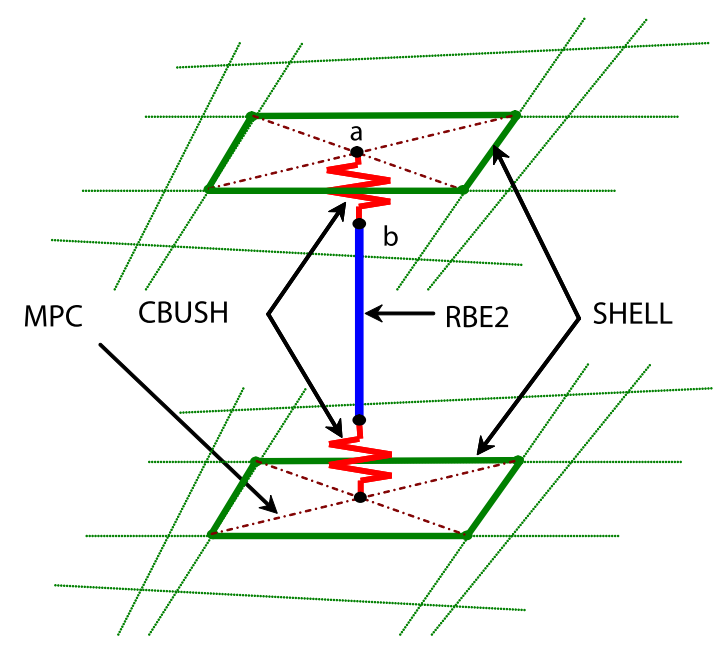

Fig. 1. Finite element model of RSW.

(spot welds and connecting components), numerical analysis computational time and ease of parameters updating.

A wide range of models have been used in the past to optimize spot welded structures, for example: an umbrella model [1], a rigid bar model [2], a beam element [5], and a rigid element combined with 3-dimensional elastic element [4]. In the present study, we are specifically interested in the modal behavior of structure which requires only the global effect of RSWs on the eigenfrequencies and eigenmodes. Towards this end, a very simple model where each spot weld is composed of two CBUSH (generalized spring and damper connection) elements and one RBE2 (rigid body element) element available in MSC.NASTRAN [11] has been used. One end of each CBUSH element is connected with the shell element using multiple point constraints (MPC) as shown in Fig. 1. The presence of a spot weld is thus be easily controlled by connecting or disconnecting the CBUSH element from the structure. Meanwhile, it should be noted that the proposed optimization procedure is in no way limited to the simple RSW models.

\section{Decision making indicators}

The decision making indicators (DMIs) are the tools implemented in the spot welding optimization procedure to select the spot welds which are redundant and should be removed or those which are critical and should be reinforced. The DMIs are used to correlate the contribution of individual spot welds to the target behavior and ideally should be easily calculated and lead to unambiguous choices. Two categories of indicators can be envisaged, namely a priori and a posteriori. The former are indicators which forecast in advance the influence of spot welds without removing them from the structure while the latter require the explicit removal of the spot welds from the structure. In practice, a posteriori indicators are very costly to evaluate since they require a large number of full model analyses. The change in elastic strain energy is an example of a posteriori indicator. Moreover, various defect detecting methodologies can be used for these indicators where removal of RSWs is interpreted as a defect. A priori indicators are generally far more efficient in terms of computational time and a number of different energy and force based indicators can readily be defined such as: elastic strain energy of RSWs, elastic strain energy of RSWs and their adjacent shell elements and forces in the RSWs.

\subsection{Description of DMIs}

In this paper, we will focus on three $a$ priori indicators and one $a$ posteriori indicator. In this section, we first define these indicators and later on, we shall illustrate their correlation with eigenfrequencies.

\subsubsection{Elastic strain energy in spot weld}

Among the four considered indicators, this is the simplest one. For the modal behavior it can be expressed as:

$I_{1}: E_{i, k}^{1}=U_{k}^{T} K^{e, i} U_{k}$

where $E_{i, k}^{1}$ is the elastic strain energy of $i$ th RSW for the mode $k, U_{k}$ is the $k$ th eigenvector and $K^{e, i}$ is the element stiffness matrix of $i$ th RSW.

\subsubsection{Elastic strain energy in spot weld and adjacent shell elements}

Shell elements joined by a given spot weld may have more influence on the dynamic behavior than the considered spot weld due to their size and stiffness, which may not allow the indicator $I_{1}$ to precisely capture the influence of the spot weld. To compensate for this effect, the energy of the surrounding shell elements is added to construct this indicator. Additionally, the energy is normalized by the volume of the adjacent shell elements in order to remove the effect of their varying sizes. This indicator can be expressed as:

$I_{2}: E_{i, k}^{2}=E_{i, k}^{1}+U_{k}^{T} K^{s h, i} U_{k} V_{m}^{s h} / V_{t o t}^{s h, i}$,

where $E_{i, k}^{2}$ is the elastic strain energy of $i$ th RSW and its adjacent shell elements for the mode $k, K^{\text {sh,i }}$ is the stiffness matrix of shell elements adjacent to $i$ th RSW while $V_{m}^{s h}$ and $V_{\text {tot }}^{s h i}$ are respectively the mean volume of shell elements adjacent to all RSWs and the total volume of the shell elements adjacent to $i$ th RSWs.

\subsubsection{Modal forces in spot weld}

For spot welds composed of CBUSH elements, the modal forces for the spot weld can be evaluated and considered as an indicator of the role of the spot weld in the energy transfer path:

$I_{3}: F_{i, k}=K^{e, i}\left(U_{k}^{b, i}-U_{k}^{a, i}\right)$,

where $F_{i, k}$ is the modal force of $i$ th RSW for the mode $k$, while $U_{k}^{a, i}$ and $U_{k}^{b, i}$ are the components of the eigenvector of mode $k$ corresponding to the node points $a$ and $b$ of $i$ th RSW (Fig. 1).

\subsubsection{Hypersensitivity energy}

This indicator is based on the work of Ouisse and Guyader [12], where it was shown that the hypersensitivity energy is closely related to the eigenfrequency shifts. Evaluating this indicator requires only the solution of a static problem. Indeed, while the required calculation cost is clearly higher than that required by $a$ priori indicator, it is still much lower than that required by a true a posteriori indicator, especially since the impact of removing several RSWs can be estimated in a single calculation. The hypersensitivity energy can thus be considered to be a quasi posteriori indicator and is given by:

$I_{4}: E_{i, k}^{3}=R_{k}^{T} K^{e, i} R_{k}$,

with $R_{k}=K_{1}^{-1}\left(K_{1}-K_{0}\right) U_{0, k}$,

where $E_{i, k}^{3}$ is the hypersensitivity energy for $i$ th RSW for the mode $k$, $R_{k}$ is the residual modal displacement for the mode $k, K_{0}$ is the stiffness matrix of the original structure, $K_{1}$ is the stiffness matrix of the structure with some missing RSWs and $U_{0, k}$ is the eigenvector of the original structure for the mode $k$.

\subsection{Illustration and discussion}

A tube-like structure assembled by 166 RSWs having approximately 46000 dofs is used to illustrate the correlation of the pro- 
posed decision making indicators to the global modal behavior due to the removal of RSWs. The structure is in free-free state and thus possesses six rigid body modes. The eigenfrequencies of the first five elastic modes ranging from $50-200 \mathrm{~Hz}$ have been considered as target behaviors. A FE model of the tube along with five considered elastic modes are shown in Fig. 2.

Each and every spot weld was removed from the tube one by one to evaluate their impact on the eigenfrequencies. Only one modal analysis is sufficient to calculate the values for indicators $I_{1}, I_{2}$ and $I_{3}$ for all spot welds while 166 static analyses (Eq. (5)) in addition to one modal analysis are required to calculate the values of indicator $I_{4}$. The eigenfrequency shift is calculated as:

$\Delta \lambda_{i}=\left(\lambda_{i}^{0}-\lambda_{i}^{1}\right) / \lambda_{i}^{0}$,

where $\Delta \lambda_{i}$ is the relative frequency shift, $\lambda_{i}^{0}$ is the eigenfrequency of the complete model and $\lambda_{i}^{1}$ is the eigenfrequency after removal of $i$ th spot weld.

The values of all indicators are separately plotted against the true eigenfrequency shifts in Figs. 3-6 for five elastic modes separately.

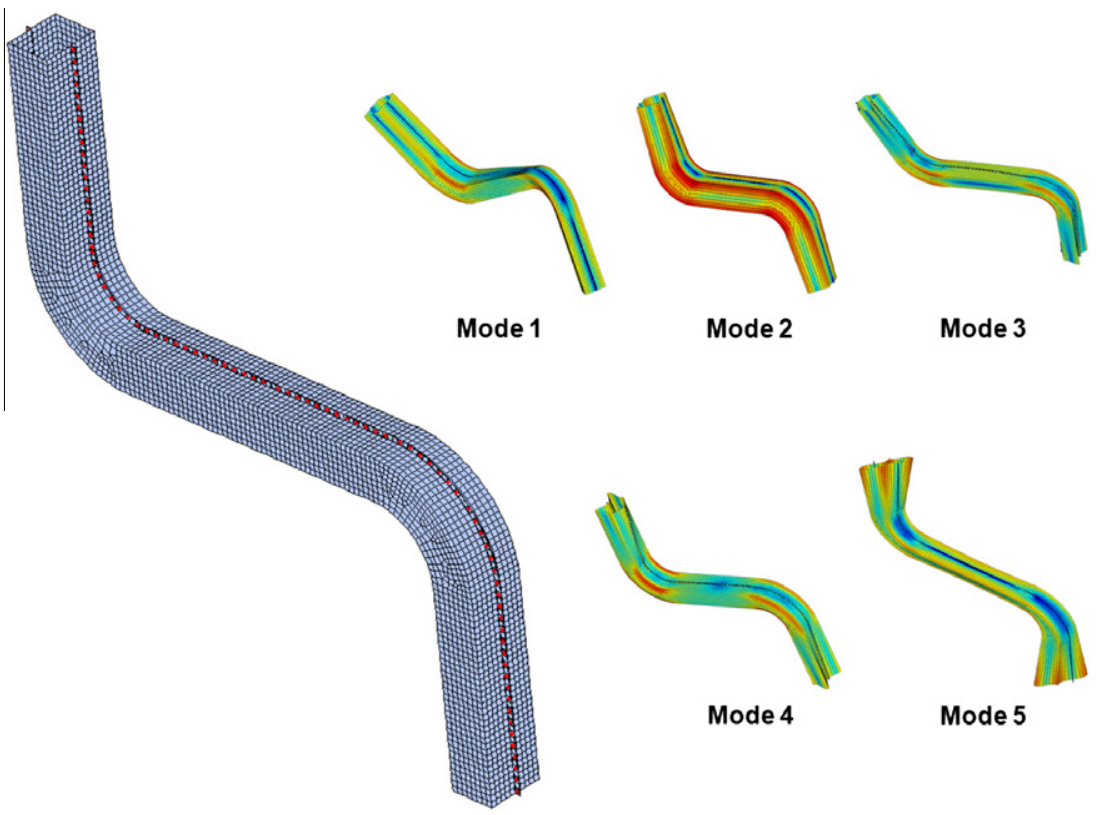

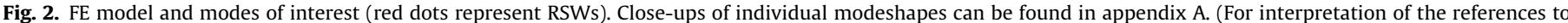
colour in this figure legend, the reader is referred to the web version of this article.)
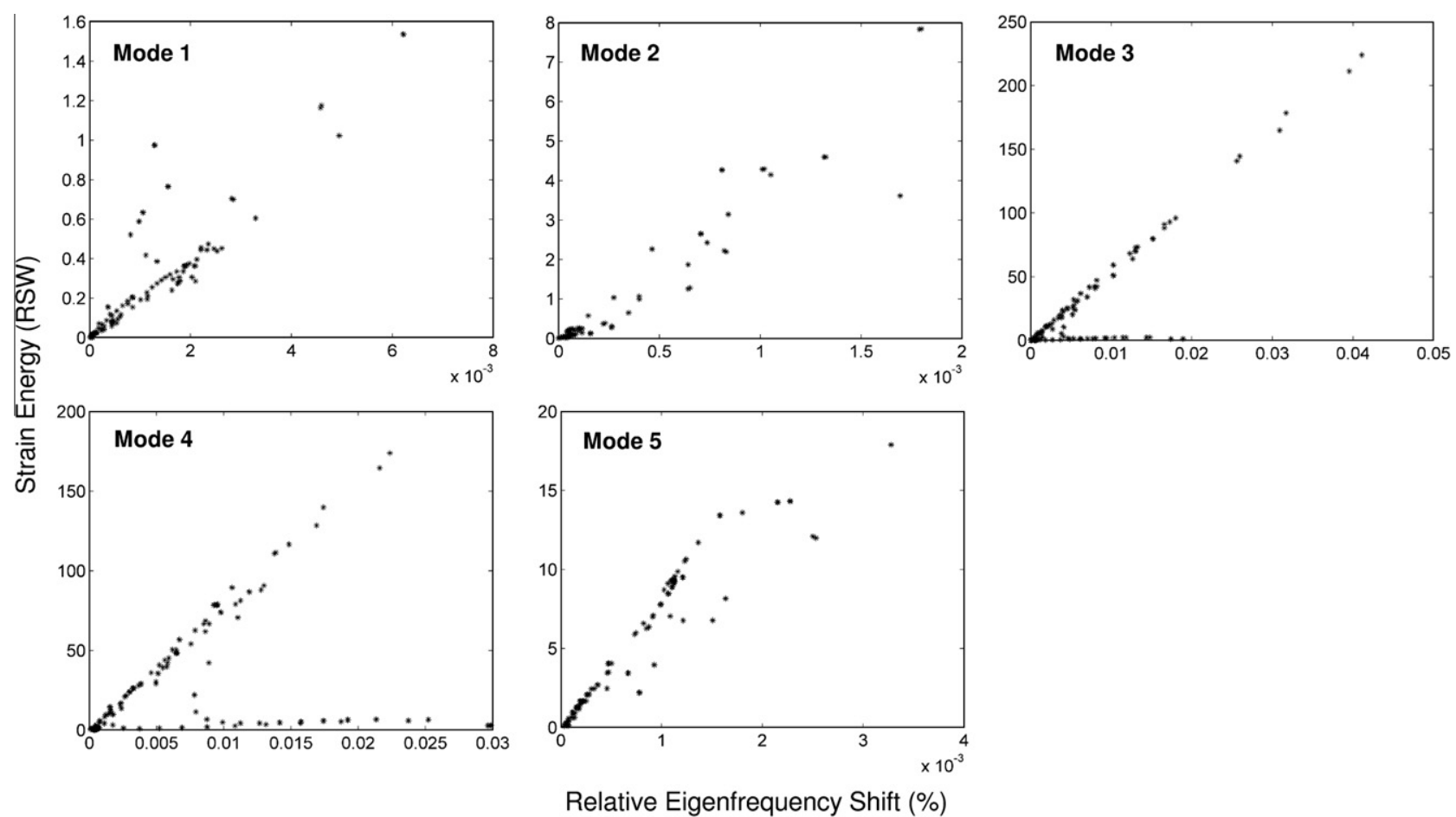

Fig. 3. Correlation of indicator $I_{1}$ with eigenfrequency shifts. 

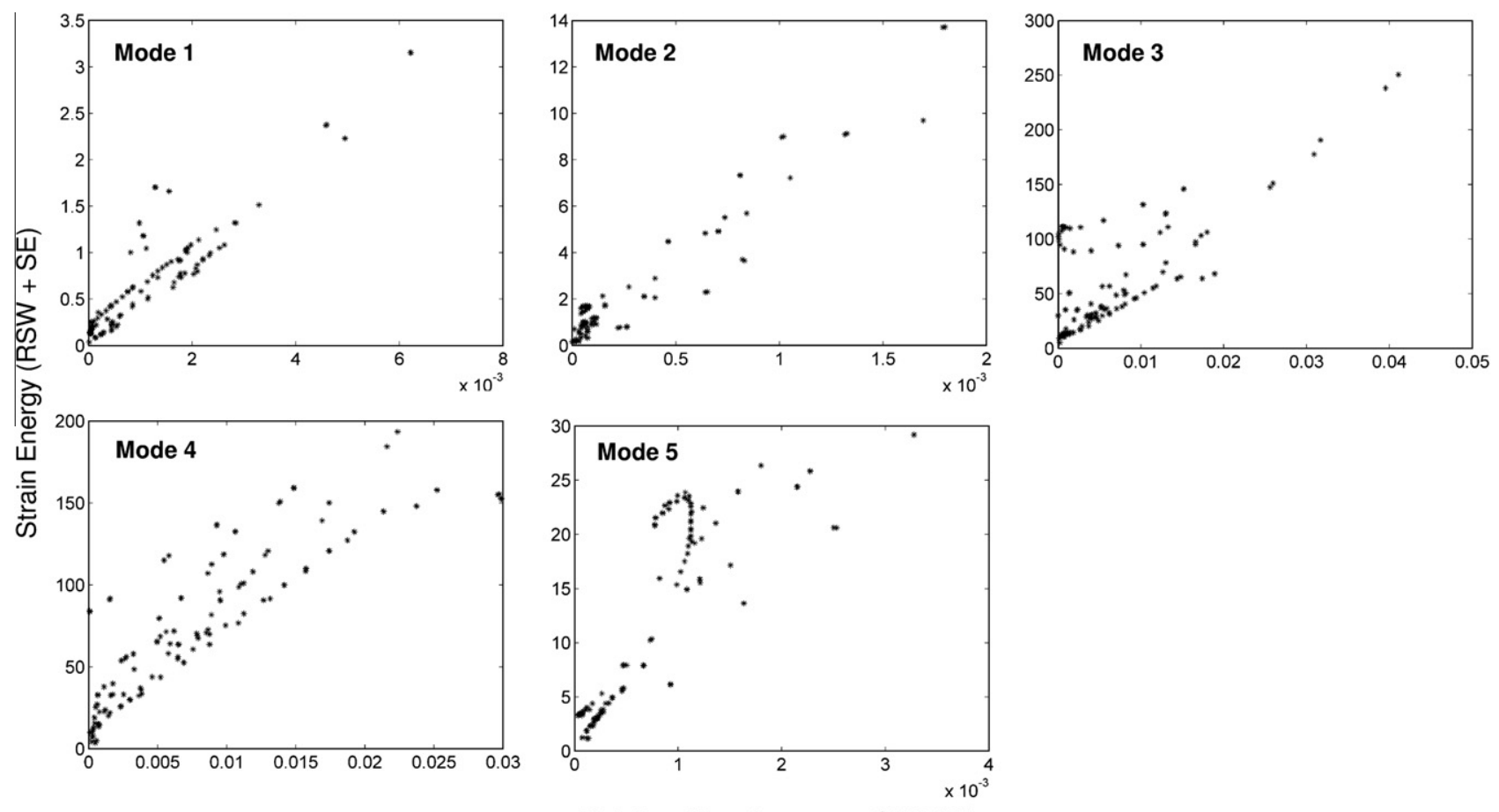

Relative Eigenfrequency Shift (\%)

Fig. 4. Correlation of indicator $I_{2}$ with eigenfrequency shifts.
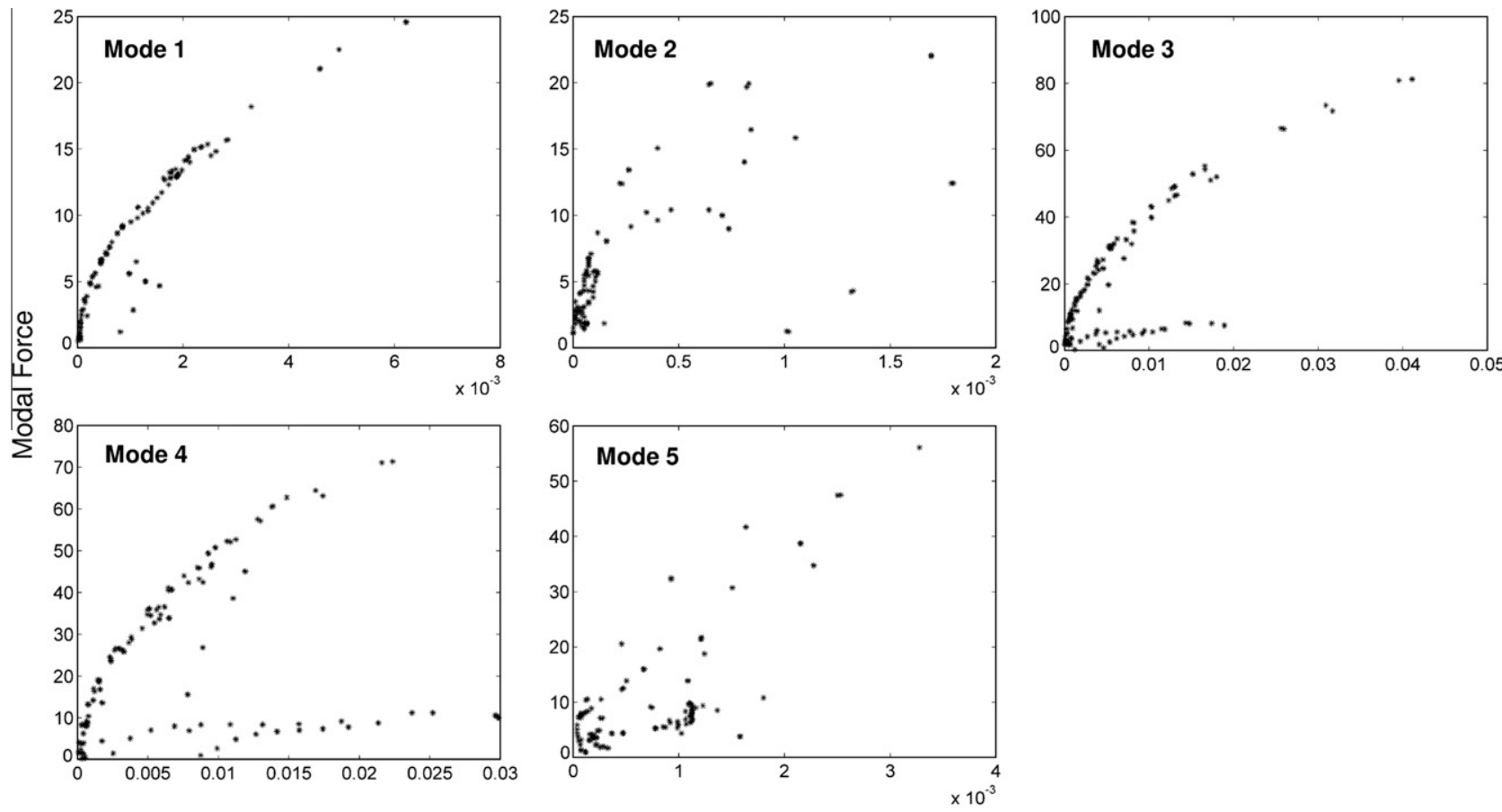

Relative Eigenfrequency Shift (\%)

Fig. 5. Correlation of indicator $I_{3}$ with eigenfrequency shifts.

The plots show that the indicators $I_{1}$ and $I_{4}$ have the same trends. Indeed, they illustrate a good correlation with the eigenfrequency shifts for modes 1,2 and 5. However, the best correlation is given by indicator $I_{4}$ for these modes, while indicator $I_{1}$ exhibits a few uncorrelated situations. This must be balanced by the higher calculation cost required by indicator $I_{4}$. However, for the remain- ing modes, although the tendencies remain good, branches of different slopes appear. One of the branches has a low slope which signifies that spot welds with lower values produce high eigenfrequency shifts in comparison to the other branch. This simple example demonstrates that there are situations where the indicator will fail. This means that if these type of indicators are used in the opti- 

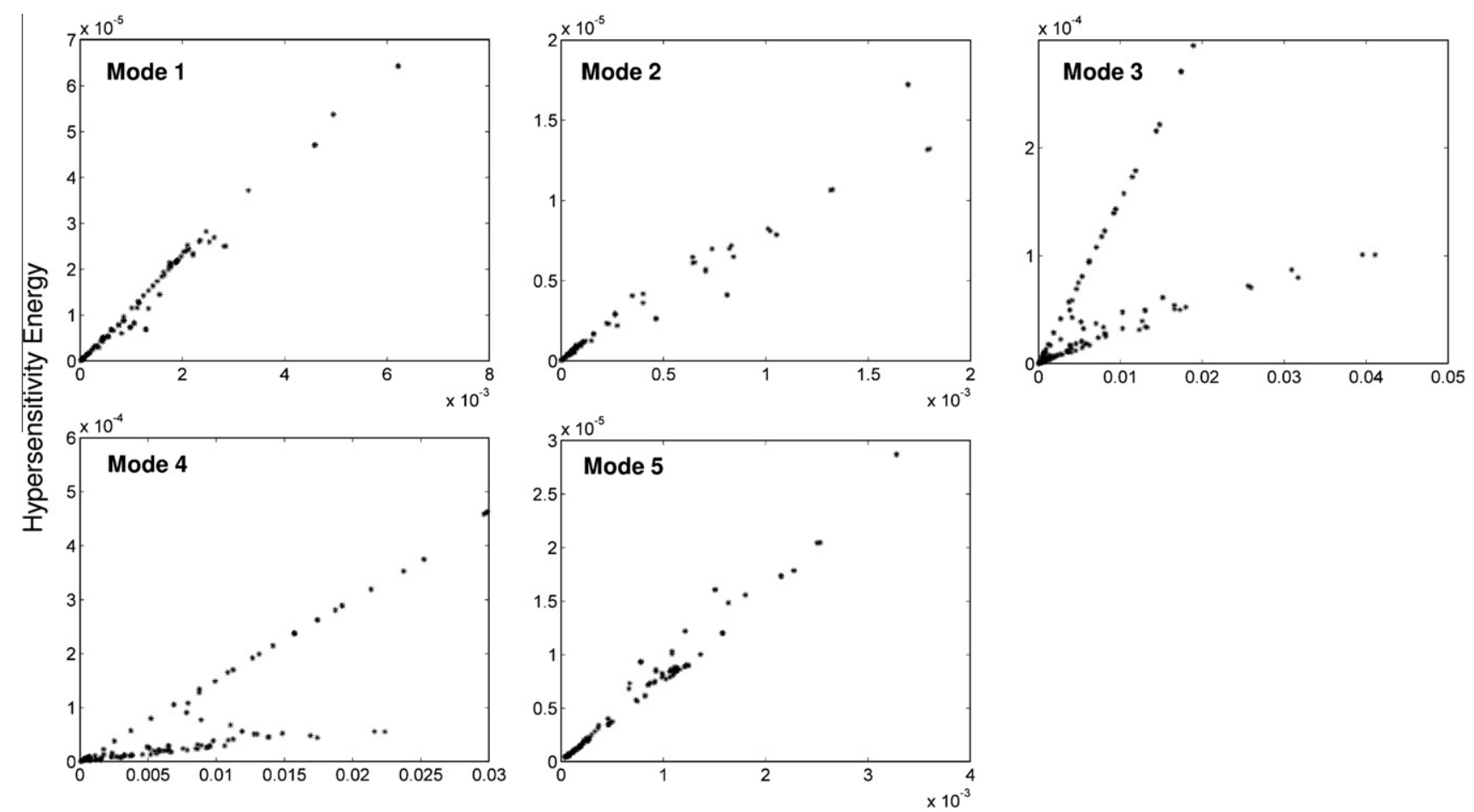

Relative Eigenfrequency Shift (\%)

Fig. 6. Correlation of indicator $I_{4}$ with eigenfrequency shifts.

mization procedure, then in these cases the procedure will fail to remove spot welds having the lowest influence. The indicator $I_{3}$ has a lower correlation and still exhibits the branches for two modes.

The results for indicator $I_{2}$ show that though the correlation is not as strong as for the other indicators, branches of different slopes do not appear. This fact makes the decision process simpler if it is used in the optimization process for ranking the spot welds. The procedure will not only have a higher tendency to select the spot welds whose removal yield small eigenfrequency shifts but also will readily identify the spot welds of higher influence.

The proposed optimization procedure focuses on the suppression or addition of a large number of spot welds at each iteration, rather than removing spot welds one by one, in order to reduce optimization cycle time. At each step of the optimization process, a set of spot welds must be identified which, when removed, will have a minimum effect on the eigenfrequencies. Indicators $I_{1}, I_{3}$ and $I_{4}$ show identical trends, however, indicator $I_{1}$ is more precise than indicator $I_{3}$ and its calculation is simpler than indicator $I_{4}$ ( $a$ quasi posteriori indicator) which requires several analyses to completely calculate the indicator values for all spot welds. Therefore, indicator $I_{1}$ is used to compare the optimization results with respect to the indicator $I_{2}$.

To roughly test the behavior, tests were conducted by removing sets of $10 \mathrm{RSW}$ at a time from different regions of indicator values for indicators $I_{1}$ and $I_{2}$. Sets $(1,2,3),(4,5,6)$ and $(7,8,9)$ were taken from the lower, medium and higher indicator values in increasing order. Fig. 7 shows the correlation between the values of indicator $I_{2}$ with the corresponding eigenfrequency shifts indicating that the spot welds associated with higher indicator values will induce higher eigenfrequency shifts and vice versa. However, in the case of indicator $I_{1}$, the eigenfrequency shifts for the sets with average indicator values are greater than the sets with higher indicator values. This result is expected for this indicator, since there exist some spot welds of low indicator values with high effect on the eigenfrequencies. Hence, using this type of indicator could limit the effi-

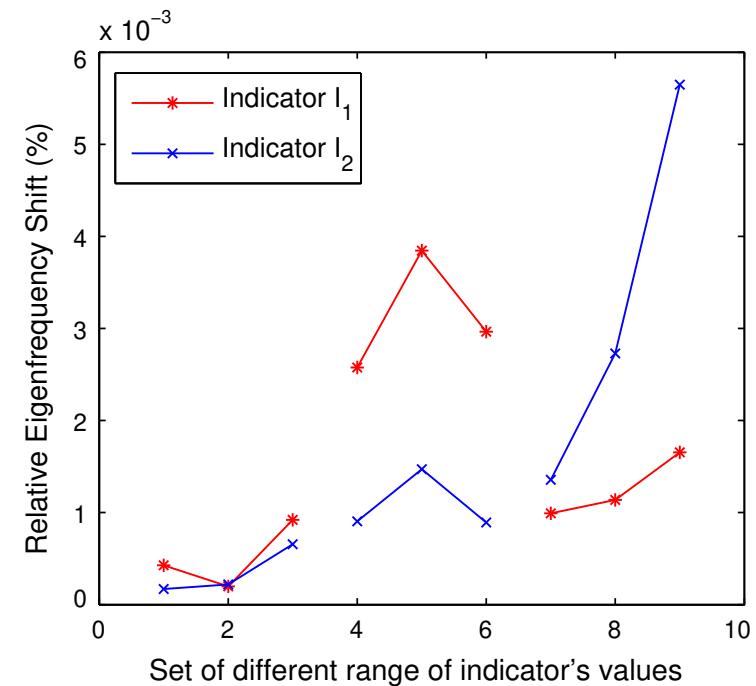

Fig. 7. Relative eigenfrequency shifts for sets of 10 RSW removed: $(1,2,3)$ - low indicator values, $(4,5,6)$ - medium indicator values, $(7,8,9)$ - high indicator values.

ciency of the optimization process by quickly degrading the eigenfrequencies. In conclusion, the indicator $I_{2}$ seems to be more reliable and is selected to be integrated in the proposed adaptive procedure.

\section{Adaptive optimization procedure}

Both types of optimization formulations discussed in Section 1 can be handled by two strategies. Ouisse and Cogan [7] solved the optimization problem using a non-adaptive procedure starting from over-populated spot welded structure. In this paper, we propose an adaptive procedure allowing spot welds to be added and 
removed throughout the optimization process. We shall apply the procedure on a full BIW for a car and shall compare the results of adaptive and non-adaptive procedures.

\subsection{Description of optimization procedure}

The proposed optimization procedure will remove the redundant spot welds from the structure and simultaneously, will add the spot welds at the sensitive locations to the proximity of the most influential RSWs. This implies that either a software is available to create new spot welds when and where needed, or that a pool of potential candidate spot welds is already available in the numerical model from which any spot weld can be activated when needed.

As all existing spot welds need to be ranked according to their contribution to the performance criteria of interest, therefore we selected the indicator $I_{2}$ to use in the procedure since it exhibits better correlation with modal behavior as discussed in Section 3 . Moreover, its effectiveness has also been demonstrated in [7].

A flowchart of the optimization procedure for modal behavior is shown in the Fig. 8 and can be summarized as follows:

1. Prepare the model and set the various procedural parameters, i.e., the number of spot welds allowed to be added/removed $(n a, n r)$, the maximum admissible eigenfrequency shift $(v)$, the

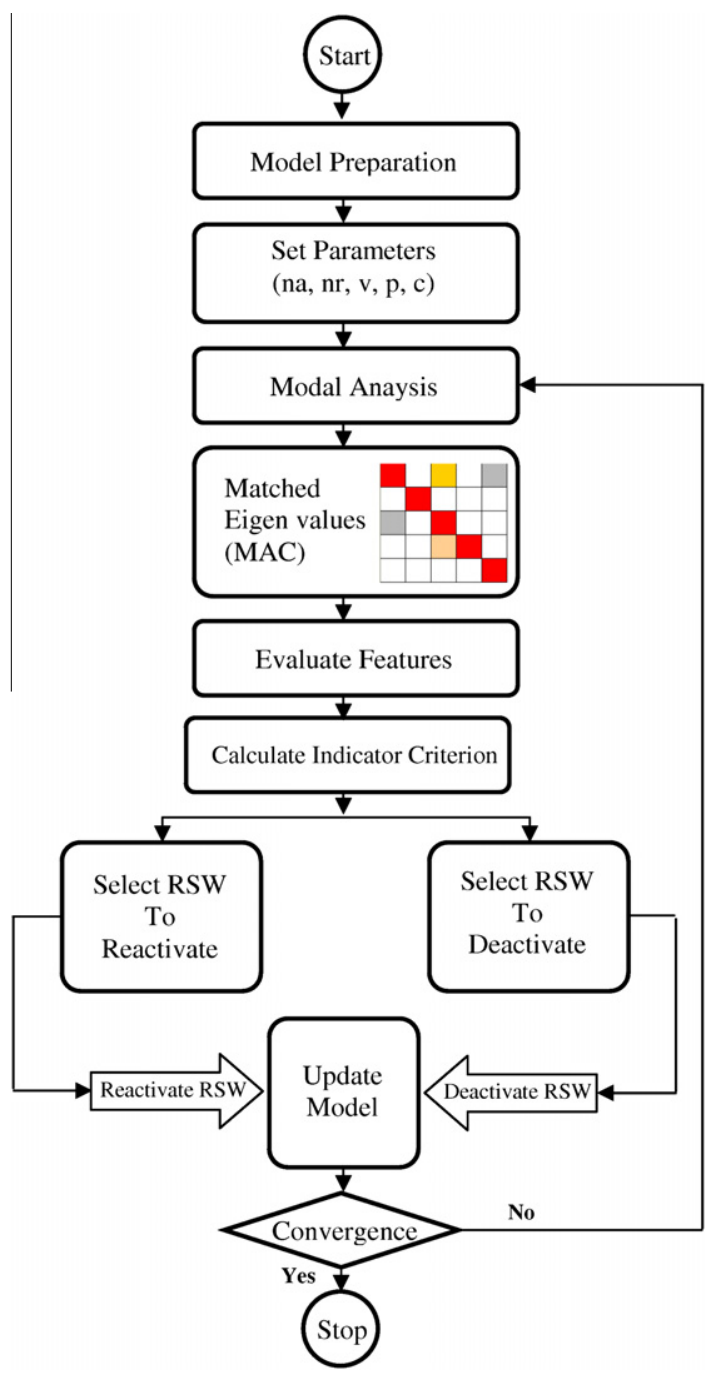

Fig. 8. Flowchart of the optimization procedure. proximity constraint value $(p)$, the maximum number of iterations $(c)$.

2. The modal analysis is performed. In order to ensure the correct matching of the eigensolutions, a modal assurance criterion (MAC) is used to compare the mode shapes of the model after removal/addition of a certain number of spot welds to the nominal model.

3. The indicator values for all spot welds are calculated.

4. Spot welds are selected for removal taking into account all imposed constraints (e.g., integrity, proximity.). See [7] for details of these constraints.

5. Candidate spot welds are selected for addition in the proximity of critical RSWs.

6. The model is updated by deactivating/reactivating the selected spot welds in steps 4 and 5 .

7. Convergence is checked. If achieved, the procedure stops otherwise return to the step 2 above.

\subsection{Application example}

The procedure is applied on a full BIW for a car to solve both types of optimization formulations which MSC.NASTRAN model having approximately $1,000,000$ dofs is shown in Fig. 9(a). The parts are assembled along 382 interfaces containing a total of 2612 spot welds. The initial spatial distribution of RSWs is shown in Fig. 9(b). This design will be referred to as the nominal design and the corresponding number of RSWs and frequencies are taken as references to calculate the relative shifts in frequencies and the increase or decrease in the number of RSWs.

The procedure requires new spot welds to be added in the structure to create a reinforced or over-populated design. Hence, we created a pool of 1494 ( $57 \%$ of the nominal design) candidate RSWs on different interfaces uniformly. Note that the RSWs removed during the optimization process will be placed in this pool and can thus be reactivated again if necessary. The performance criteria in this example is based on the eigenfrequencies of the first torsion and bending modes (see Fig. 10). MSC.NASTRAN is used to perform the modal analysis up to $65 \mathrm{~Hz}$ and takes almost $20 \mathrm{~min}$ on a Windows XP professional based computer having processor speed of $3.0 \mathrm{GHz}$ with $2.0 \mathrm{~GB}$ RAM.

\subsubsection{Case I: performance optimization}

In this case, the goal is to maximize the eigenfrequencies of the first torsion and bending modes with a predefined number of RSWs, that is to say, the total number of RSWs is constant and set to 2612. The problem here is thus to find the best distribution of a fixed number of RSWs by relocating them so that the contribution of each RSW will be maximum for the targeted eigenfrequencies.

In this example, the value of proximity constraint was set to 3 . This means that two spot welds selected for removal should be separated by at least two spot welds in the current iteration. The number of spot welds allowed to be added and removed was set to 75 in each iteration while maintaining the total number of RSWs constant. Moreover, two modes were considered to be matched if the MAC value was more than 0.7. To maintain the integrity of the interfaces, the spot welds at either end were not allowed to be removed. However, they were taken into account when the procedure identified the critical spot welds for selecting the RSWs to add in their proximity.

Fig. 11 shows the evolutions of both frequencies for this case and the final distribution of RSWs is shown in Fig. 12. The maximum values of both eigenfrequencies are achieved in the 7th iteration. Increases of a $1.05 \%$ in frequency of mode 1 and a $0.39 \%$ in frequency of mode 2 are seen. 


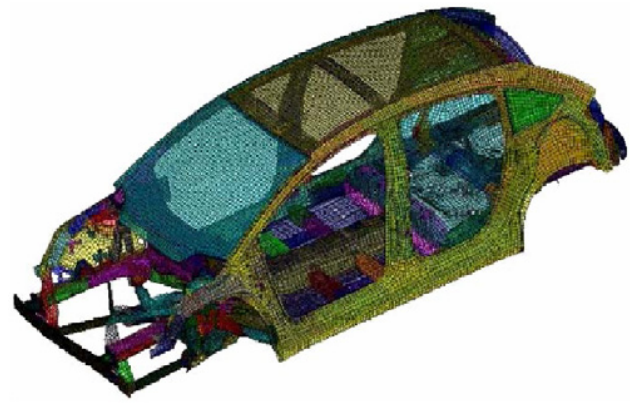

(a) Finite element model.

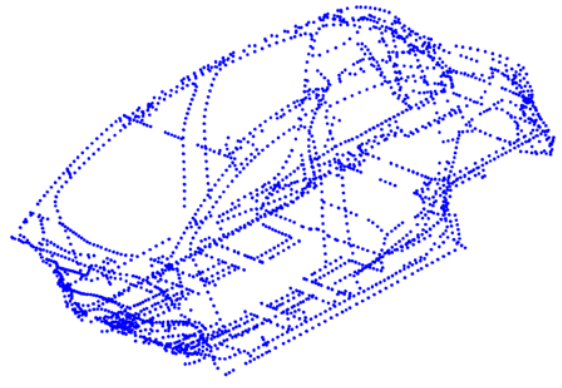

(b) Initial distribution.

Fig. 9. FE of BIW for a car and initial distribution of spot welds.

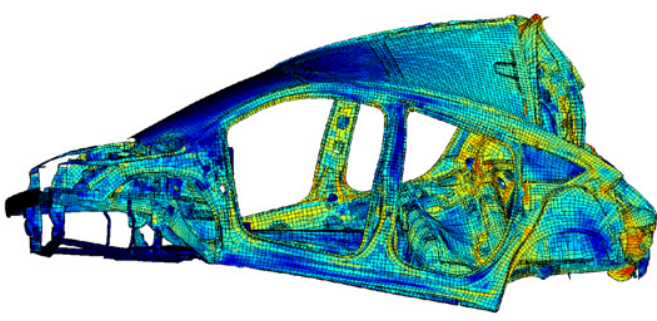

(a) Torsion mode.

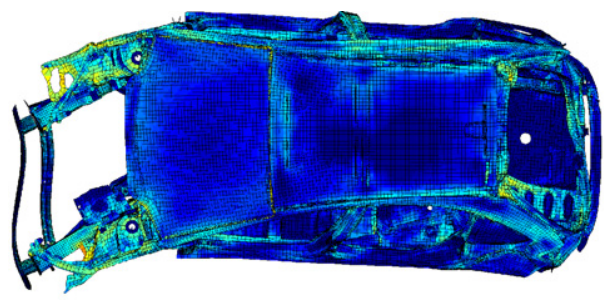

(b) Bending mode.

Fig. 10. Modes shapes of the car.

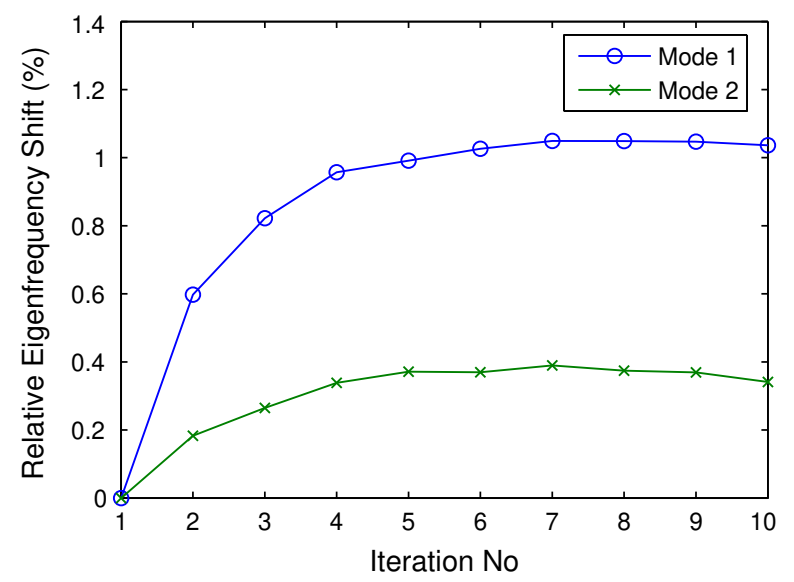

Fig. 11. Case I - optimization run for adaptive procedure.

It could also be noted that most of the improvement in both frequencies were obtained in the first three iterations while in higher iterations, no significant increases were observed. This is due to the availability of a limited number of candidate RSWs to add at the critical interfaces. We also noted that in the last iterations both frequencies start decreasing. This trend is due to the fact that some interfaces with spot welds having low indicator values were depleted in the course of optimization. As a result, few parts were loosely connected with the main assembly, thus leading to decrease in eigenfrequencies. The removal of consecutive RSWs (green color spots) can clearly be seen in Fig. 12. In practice, the optimization process should be stopped before this phenomenon occurs.

To compare the results, the optimization was also performed with the non-adaptive procedure [7]. Recall that this procedure requires an over-populated spot welded structure. Hence, we acti-

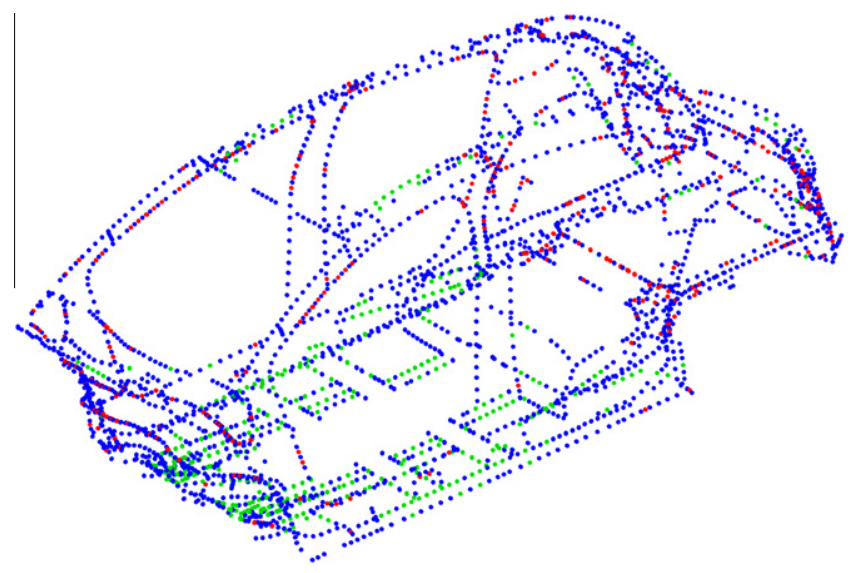

Fig. 12. Final distribution of RSWs for case I (blue: retained, green: removed, red: added). (For interpretation of the references to colour in this figure legend, the reader is referred to the web version of this article.)

vated all of the 4106 RSWs available spot welds. For this procedure, the threshold value which controls the number of spot welds selected for removal at each iteration was taken to be $1.25 \%$ while other parameters took the same values.

The non-adaptive procedure took 7 iterations to remove the extra 57\% RSWs and the evolutions of both frequencies are shown in Fig. 13 . Here, $1.02 \%$ gain in frequency of mode 1 and $0.31 \%$ gain in frequency of mode 2 was obtained. Increases in both eigenfrequencies obtained by adaptive and non-adaptive procedures are compared in Fig. 14. Results show that gain in both frequencies obtained by adaptive procedure is slightly more than to those of the non-adaptive procedure while both procedures took the same computational effort. Note that large numbers of spot welds were removed in early iterations in comparison to the final iterations. The proximity constraint dictates how the spot welds should be 


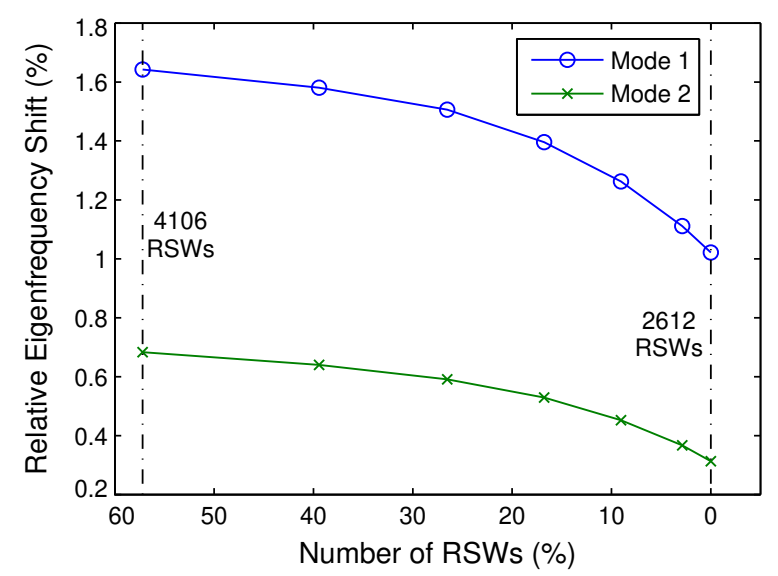

Fig. 13. Case I - optimization run for non-adaptive procedure (values on $x$-axis show the number of RSWs more than nominal model).

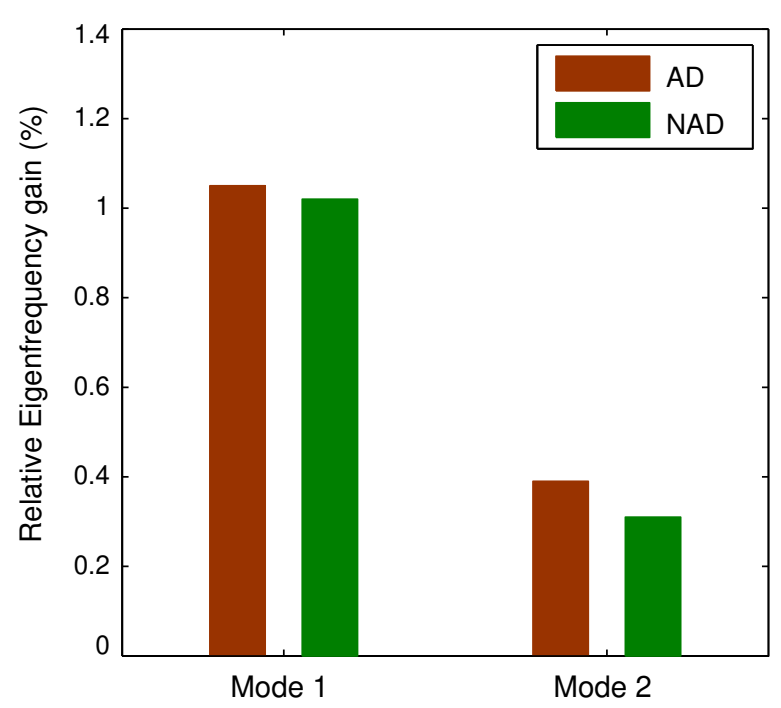

Fig. 14. Comparison of gain in frequencies for the adaptive (AD) and non-adaptive (NAD) procedures for case I.

selected for removal, therefore when large numbers of RSWs have to be removed in one iteration, the procedure is forced to select some spot welds having a higher influence due to the proximity constraint. Consequently, it has an unfavorable impact on the overall performance of the non-adaptive procedure.

\subsubsection{Case II: quantity optimization}

In this case, the objective is to minimize the number of RSWs while keeping the first torsion and bending eigenfrequencies higher than those of the nominal design.

For this case, the same procedural parameters were used for the adaptive procedure except that the number of RSWs that can be removed needs to be more than the number of RSWs that can be added. The procedure was thus allowed to remove twice as many RSWs as it can add.

Fig. 15 shows the optimization run for this case and the final distribution of the RSWs is shown in Fig. 16. The procedure efficiently removed more than $14 \%$ of the total RSWs in only 6 iterations while both eigenfrequencies of the final design are better than the frequencies of the nominal design: $0.61 \%$ higher for mode 1 and $0.1 \%$ higher for mode 2 . Fig. 15 shows that the eigenfrequencies continue to improve despite of the fact that the total number

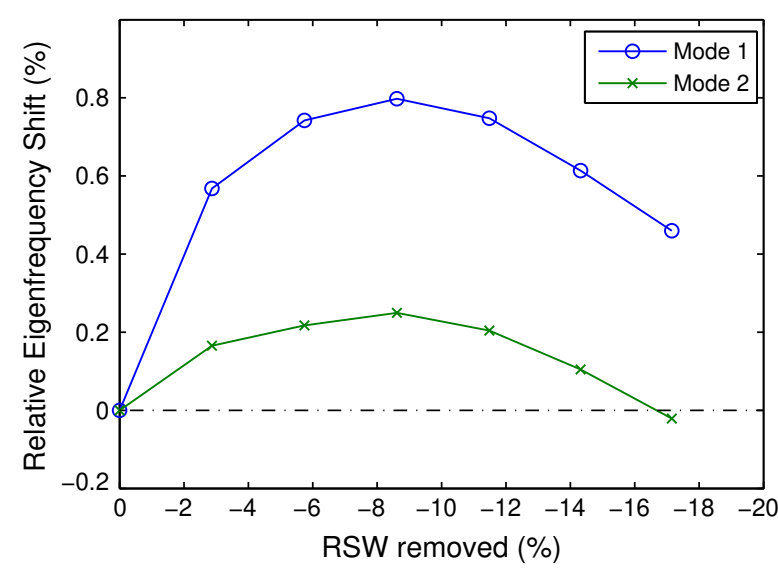

Fig. 15. Case II - optimization run for adaptive procedure (values on $x$-axis show the number of RSWs less than nominal design).

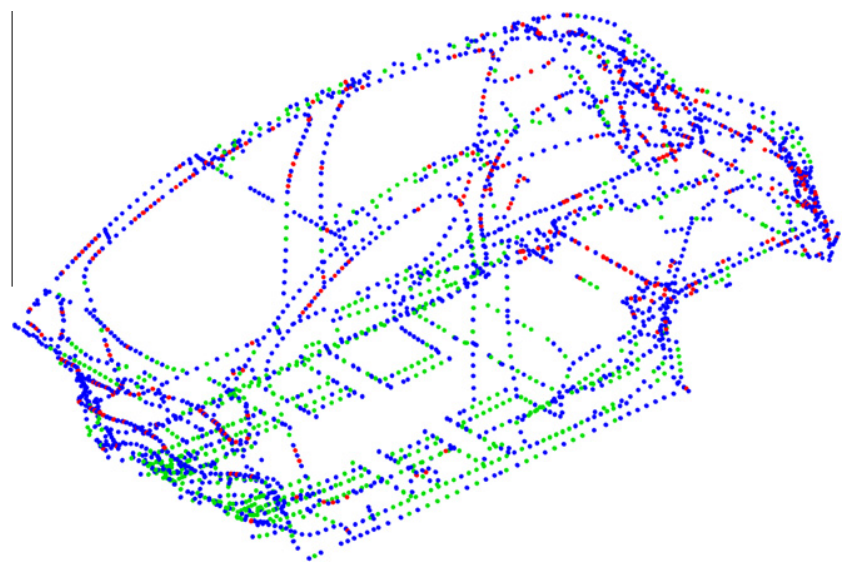

Fig. 16. Final distribution of RSWs for case II (blue: retained, green: removed, red: added). (For interpretation of the references to colour in this figure legend, the reader is referred to the web version of this article.)

of RSWs is decreasing. This is once again due to the addition of RSWs at the critical interfaces having higher influence.

As before, to compare the results an optimization was also performed with the non-adaptive procedure. Here again, procedure started from the structure containing 4106 RSWs. To properly compare the performance, the threshold value was set to $2.5 \%$ while the remaining parameters took the same values as for the adaptive procedure.

Fig. 17 shows the optimization run for the non-adaptive procedure as well as the adaptive procedure. The non-adaptive procedure was able to reduce the number of RSWs by more than $13 \%$ in 6 iterations while both the target eigenfrequencies of the final design are better than the frequencies of the nominal design. However, the adaptive procedure is able to reduce by $1 \%$ more spot weld count which is quite significant considering the large number of spot welds used to assemble a full BIW for a car.

\subsection{Discussion}

The eigenfrequencies are considered as a measure of the dynamic stiffness of the structure and an increase in the frequencies translates an increase in the structural stiffness. Note that it is the stiffness of sub-assemblies which are the main sources of overall stiffness in the structure while the contribution of the spot welds is significant up to a certain number beyond which contribution becomes negligible [13]. 


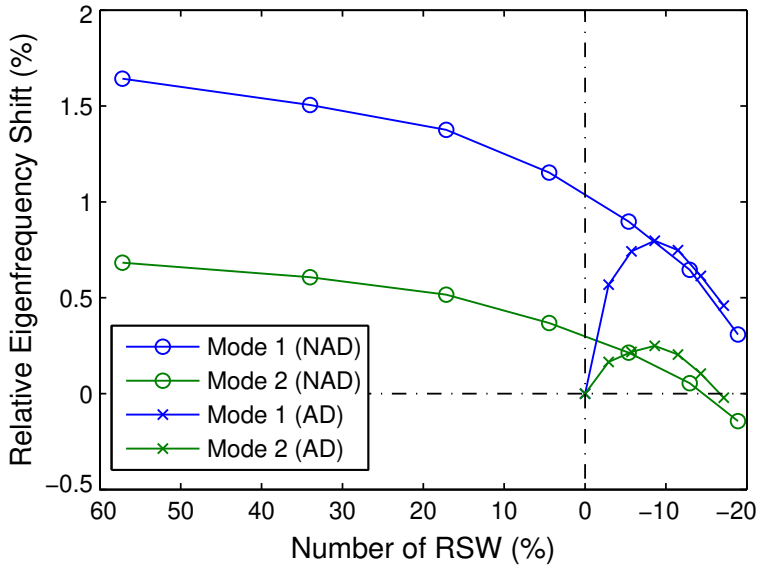

Fig. 17. Comparison of the adaptive (AD) and non-adaptive (NAD) procedures for case II (positive/negative values on $x$-axis show the number of RSWs more/less than nominal design).

Table 1

Summary of all designs.

\begin{tabular}{lllllll}
\hline Design description & $\begin{array}{l}\text { Number of } \\
\text { RSWs }\end{array}$ & \multicolumn{2}{l}{ Relative gain (\%) } & & \multicolumn{2}{l}{$\begin{array}{l}\text { Optimality } \\
\text { indicator }\end{array}$} \\
& & Mode 1 & Mode 2 & & Mode 1 & Mode 2 \\
\hline Nominal (D1) & 2612 & - & - & & 0.0155 & 0.0199 \\
Over-populated (D2) & 4106 & 1.643 & 0.683 & & 0.0100 & 0.0128 \\
Optimal case I (D3) & 2612 & 1.050 & 0.390 & & 0.0157 & 0.0200 \\
Optimal case II (D4) & 2238 & 0.610 & 0.100 & & 0.0182 & 0.0233 \\
\hline
\end{tabular}

This effect can be observed in the Table 1 where we see that an optimized design D3 can perform nearly as well, as the over-populated design D2 with a much smaller number of spot welds. This means that addition of $57 \%$ spot welds in the optimized design D3 to build design D2 are now responsible for only a $36 \%$ and $42 \%$ of the total increase in eigenfrequencies for mode 1 and mode 2 respectively. This confirms that beyond a certain number of optimally located RSWs, the addition of more RSWs in the structure has minute contribution in the eigenfrequencies. This indicates that an adequate compromise between redundant RSWs and assembly cost must be searched.

In order to properly rank the designs with different distributions and numbers of the RSWs, an indicator is defined which measures the average contribution of the RSWs for the criteria of

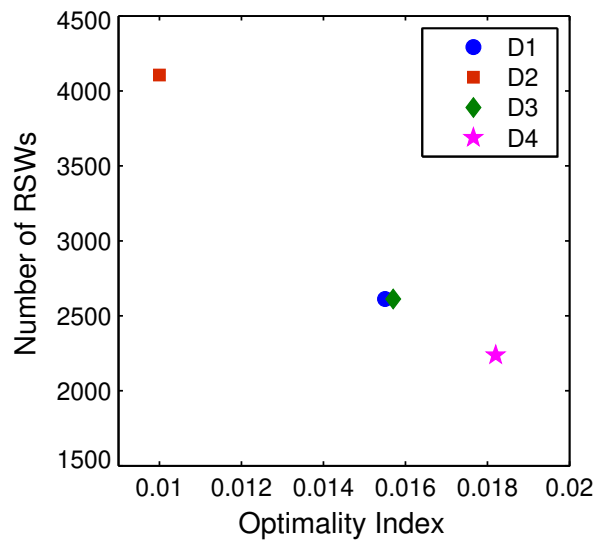

(a) Mode 1 interest. This indicator could conveniently be used to rank the different solutions:

Optimality indicator $=\frac{\text { Eigenfrequency value }}{\text { Total number of RSWs }}$.

The optimality indicator values of both modes for all four designs are mentioned in the Table 1 and can be visualized in Fig. 18 for both modes. Notice that although the over-populated design D2 has the highest eigenfrequencies, according to the indicator it is the worst solution due to presence of a large number of redundant RSWs. Moreover, despite the fact that optimized design D3 has higher eigenfrequencies due to the better distribution of the RSWs, in terms of optimality indicator, the gain is small compared to the nominal design D1. This indicates again the over abundance of redundant RSWs. Finally, the optimality indicator values for optimized design D4 are higher and demonstrate that each spot weld is adequately loaded while keeping the number of redundant spot welds to a minimum. Therefore, the design D4 could be regarded as an optimal one in terms of frequencies and total number of RSWs.

In conclusion, while the optimization of spot welded structures could be performed with a fixed number of RSWs, the gain in terms of optimality indicator is minute due to the existence of a large number of redundant RSWs. This fact motivates the use of the formulation where the number of RSWs should also be considered as variable in addition to their distribution in order to obtain a near optimal design.

\section{A posteriori robustness analysis methodology}

In this section, an info-gap robustness analysis [14] is performed to study the impact of missing RSWs on system performance. The info-gap uncertainty is modeled as:

$\mathscr{N}(\alpha, \widetilde{N})=\{N:|N-\widetilde{N}| \leqslant \alpha\}, \quad \alpha \geqslant 0$,

where $N$ and $\widetilde{N}$ are respectively the effective and nominal numbers of RSWs. Here the interpretation of the horizon of uncertainty $\alpha$ is simply the number of missing spot welds. The corresponding robustness function with respect to the $v$ th structural eigenfrequency (f) is:

$R_{v}=\max _{N \in \mathcal{N}(\alpha, \widetilde{N})}\left|\frac{f_{v}-\tilde{f}_{v}}{\tilde{f}_{v}}\right|$.

In other words, the robustness is defined here as the worst case performance among all possible design configurations consistent with a given level of uncertainty in terms of missing spot welds. Hence,

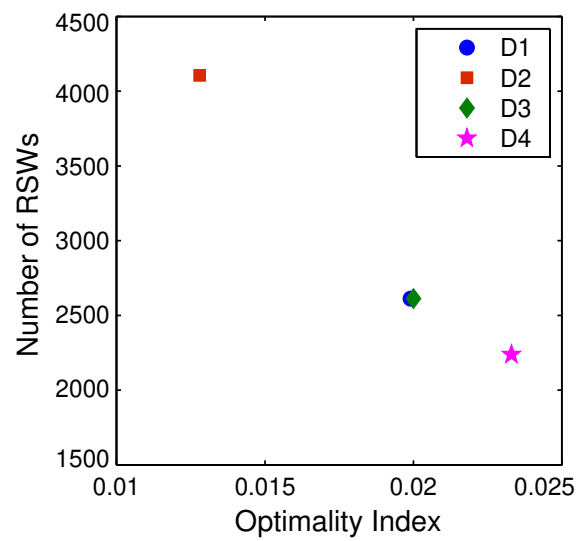

(b) Mode 2

Fig. 18. Optimality indicator vs number of RSWs for four designs. 
design A will be considered more robust than design B if the worst case variation in performance due to the absence of a specific number of spot welds for design A is less than that of design B. Alternatively, a design is considered more robust when it can afford to lose a greater number of the most influential spot welds without compromising a specified critical performance limit.

As discussed earlier, while a Monte Carlo simulation is a potentially straight forward way to measure the impact of missing RSWs on a spot welded structure, the large number of analyses required to obtain converged statistics renders this approach infeasible in the present context. Hence, we propose a simple and less costly approach based on an examination of the impact of the most influential spot welds on the performance criteria of interest. In Section 3, it has been shown that spot welds with larger indicator values have a relatively higher impact on the eigenfrequencies than the spot welds with smaller indicator values. The robustness function will be approximated here by progressively removing the most influential RSWs. Moreover, an examination of this robustness curve will naturally define a set of critical spot welds that should either be quality controlled or reinforced. This curve can also serve as a useful tool for deciding how many spot welds should be inspected after assembly while taking into account the total number of RSWs and a desired robustness level.

\subsection{An iterative procedure to obtain the robustness curve}

The proposed iterative procedure (IP) to obtain the robustness curve for the modal behavior can briefly be described as follows:

1. All existing spot welds are ranked according to decreasing value of the indicator criteria,

2. a predefined number of spot welds of higher ranks are selected for removal while taking into account few assumptions mentioned later on,

3. an analysis is performed to evaluate the impact of removed spot welds on the modal behavior,

4. the indicator criteria is calculated for the remaining spot welds,

5. stop, if stopping criteria is met, otherwise, go to the first step above.

Although each spot weld is assumed to have an equal chance of being either defective or missing, in practice the presence of a large number of spot welds can be ensured automatically by careful implementing the spot welding process. Moreover, to remain as realistic as possible we make the following assumptions:

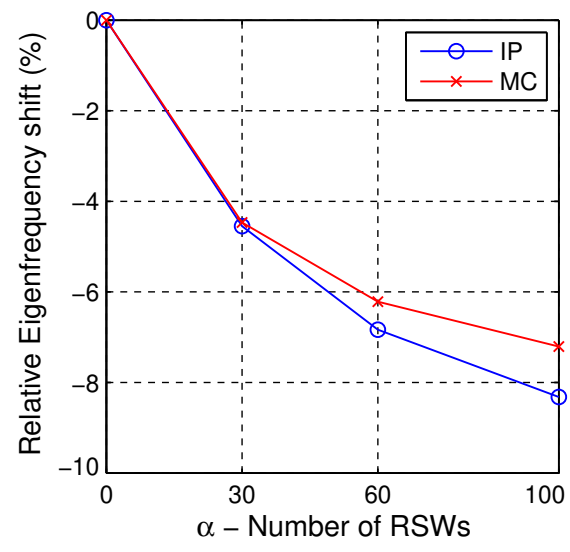

(a) Mode 1
- the welding process is assumed to be designed in such a manner that the integrity of the RSWs located at either end of each interface are ensured automatically (i.e., zero chance of being defective or missing),

- for each interface, not more than $10 \%$ of the spot welds may be defective or missing (various percentage for each interface may be considered taking into account different factors such as access to the locations, sizes and number of RSWs, etc.),

- at least one spot weld may be defective or missing from each interface but the spot welds on interfaces with 4 RSWs or less are assumed to be effectively present,

- finally, two consecutive spot welds can not be defective or missing.

\subsection{Verification of iterative procedure}

A MC simulation with a strain weighted scheme [7] is used to illustrate the effectiveness and efficiency of the proposed iterative procedure (IP) to obtain the worst case degradation robustness curves.

Three MC simulations with 75 samples each have been performed to simulate the loss of respectively 30, 60 and 100 spot welds with respect to the nominal design. The robustness curve is then obtained by taking the maximum variation observed in each simulation for both target modes. Meanwhile, we also used the iterative procedure (IP) to obtain the robustness curves, wherein 10 spot welds having the largest indicator values are removed at each iteration.

The robustness curves obtained by MC simulations as well as the IP are plotted in Fig. 19. For 30 missing spot welds, the worst case variations in eigenfrequencies obtained by the MC simulation are comparable to those of proposed iterative procedure (IP) but the MC simulation is much more costly: 75 analyses for the MC simulation compared to 3 analyses for the IP. Meanwhile, for 60 and 100 missing spot welds, the MC simulation is unable to match the IP and suggests that as the number of missing spot welds increases, the MC simulation will require a larger number of samples to produce comparable results to those obtained by the IP. The proposed iterative procedure is not only able to find a reasonably realistic worst case scenario but is also highly efficient in terms of calculation time: 10 analyses for 3 levels of missing of spot welds compared to 225 analyses for MC simulation.

\subsection{Illustration: robustness analyses}

We applied the iterative procedure (IP) on the four designs (D1, D2, D3 and D4) to obtain the robustness curves for up to 100 miss-

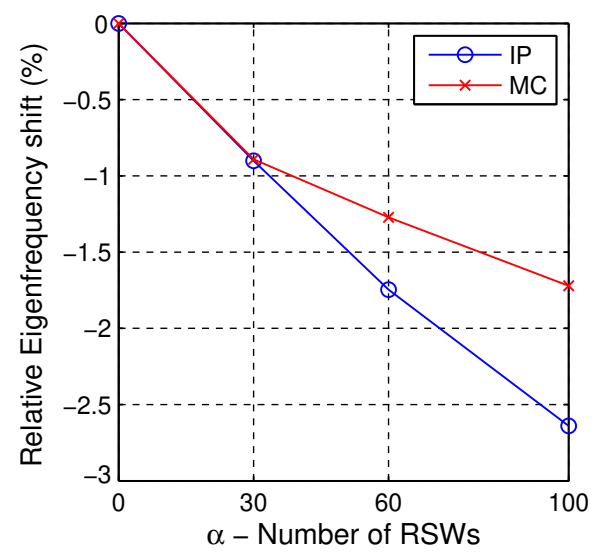

(b) Mode 2

Fig. 19. Robustness curves obtained by IP and MC for design D1. 
ing RSWs by removing 10 spot welds of highest indicator values in each iteration. The number of RSWs removed in each iteration may be increased or decreased by considering the trade-off between the total number of spot welds to be checked and the time required for a numerical analysis.

The robustness curves for both modes are shown in Fig. 20 for all four designs. Once again, the eigenfrequencies of the nominal design D1 were taken as references to calculate the relative frequency shifts for all designs. The curves illustrate that the influence of the number of missing spot welds increases with the reduction in the total number of RSWs used to assemble the structure but the degradations observed in eigenfrequencies are far less when compared to the reduction in the total number of spot welds. The optimized design D3 is more robust than design D1, though both have the same number of RSWs. Similarly, the optimized design D4 is also less sensitive than the design D1 for an increasing number of missing RSWs despite the fact that design D4 has 15\% less number of spot welds.

Once again, a design is considered to be more robust when it can tolerate the loss of more spot welds before attaining a specified critical level of performance. For example, design D2 can tolerate the loss of 70 of the most influential RSWs before the degradation in an eigenfrequency exceeds $5.0 \%$ for mode 1 while the designs D3 and D4 can only tolerate the loss of 50 and 30 spot welds under the same conditions. This clearly illustrates the trade-off between the cost of a design with a given number of spot welds and its robustness to missing spot welds.

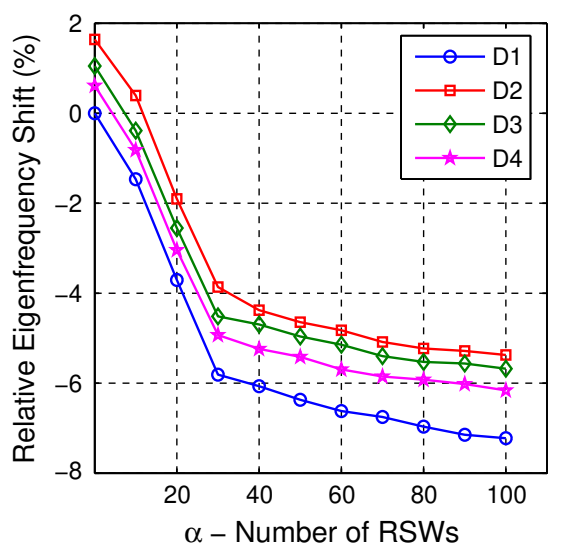

(a) Mode 1
However, note that most of the eigenfrequency degradations are due to only the first 30 RSWs for all designs. This implies that these are the most critical RSWs and need special attention from the designer. Indeed, this proves to be valuable information that can be used to improve the design in various ways, for example:

- design of a small number of critical spot welds can be modified to improve their performance characteristics,

- subparts joined by the critical spot welds can be redesigned to absorb their adverse impact,

- most critical spot welds may be quality controlled to ensure their effective presence.

\section{Quality control in spot welded structures}

We have seen that, following the optimization, there may still remain some spot welds whose absence can cause large variations in the performance criteria. Hence, to take the advantage of information obtained from the robustness curves, we propose to introduce a quality control (QC) of a limited number of spot welds in order to guarantee the acceptable robustness of the population of identical structures due to the absence of a remaining set of uncontrolled spot welds.

To demonstrate this, we assumed that the first 20 spot welds identified while obtaining the robustness curves were quality controlled for all designs. The iterative procedure (IP) was applied again to obtain the robustness curves for the remaining uncon-

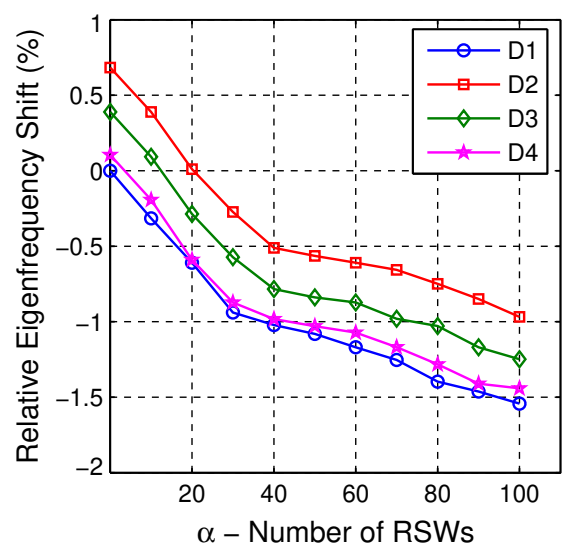

(b) Mode 2

Fig. 20. Robustness curves for two modes for four designs.

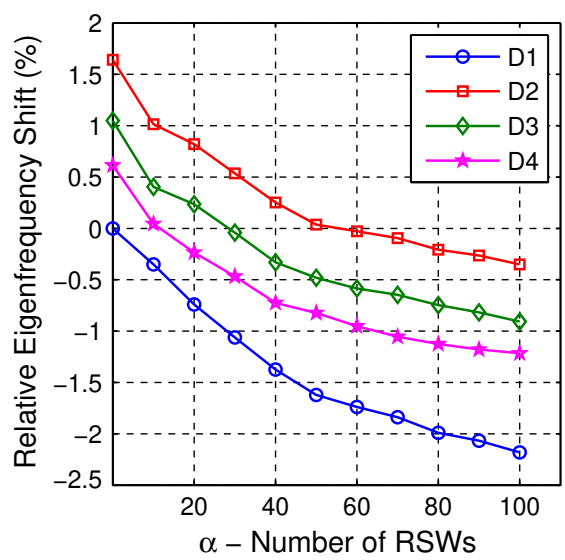

(a) Mode 1

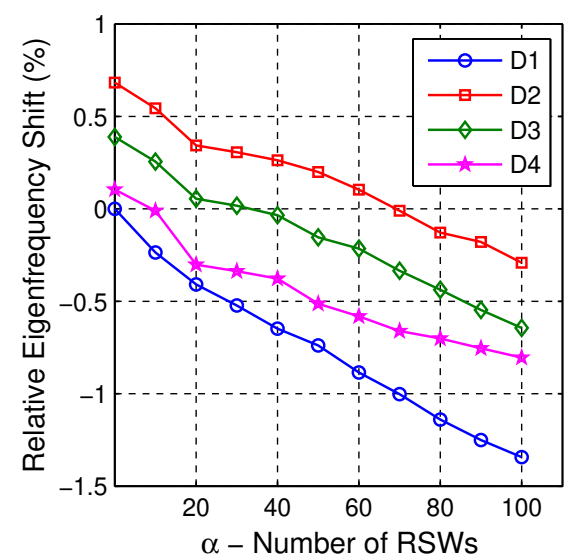

(b) Mode 2

Fig. 21. Robustness curves for two modes after quality control of 20 RSWs. 
trolled spot welds and new robustness curves obtained are plotted in Fig. 21 for both modes. These curves show the remarkable improvement in the robustness (i.e., up to 3 times) due to missing of the most influential spot welds from the remaining uncontrolled spot welds while the trends remain the same among all four designs.

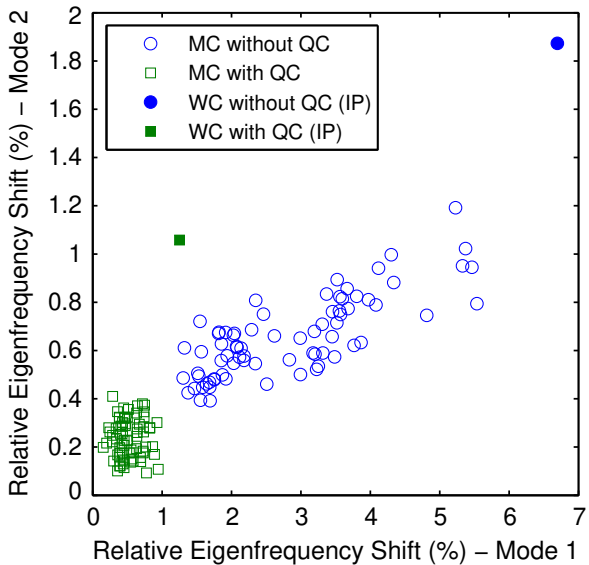

(a) Scatter plot with and without QC

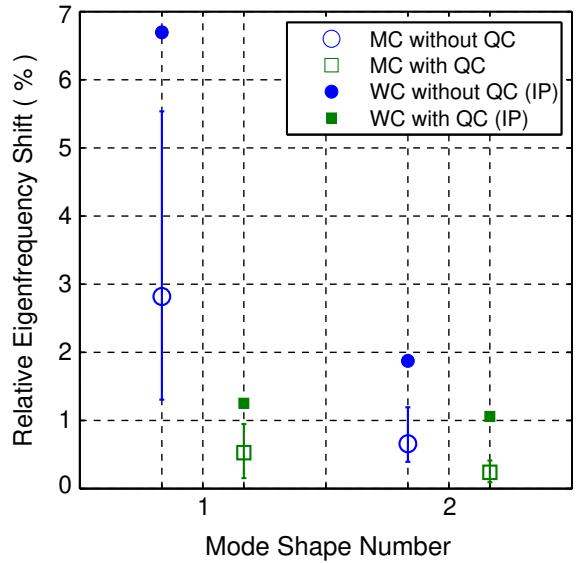

(b) Statistical data plot with and without QC (min, mean, max)

Fig. 22. MC simulations - effect of QC on robustness for design D3.

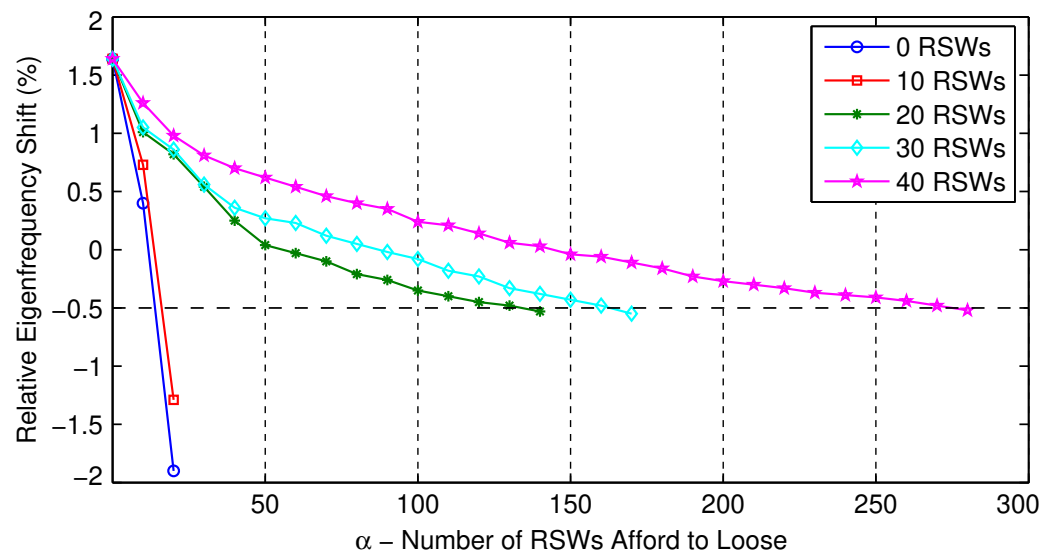

Fig. 23. Effect of increasing number of quality controlled RSWs.

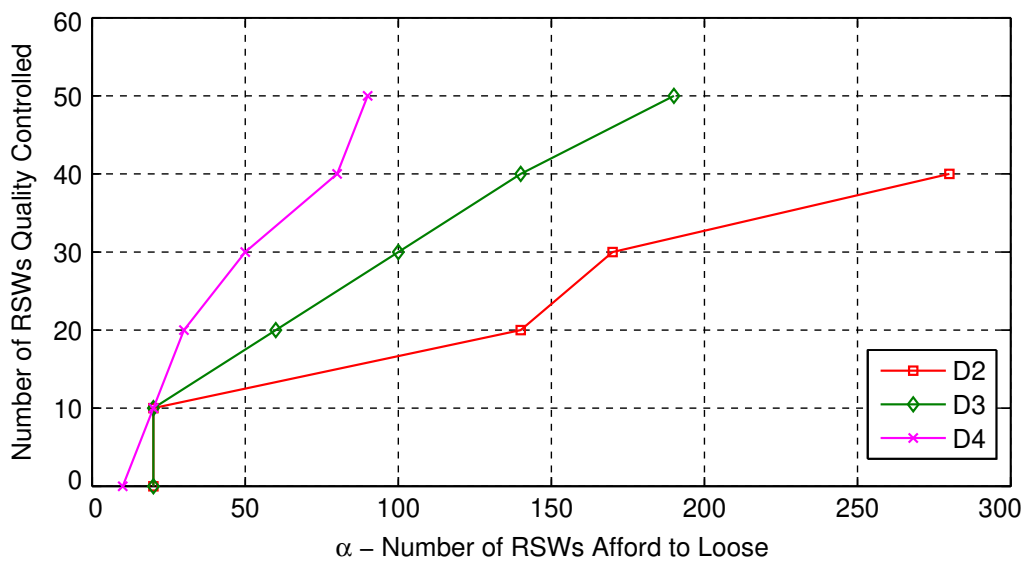

Fig. 24. Trade-off curves to select the number of RSWs to be quality controlled. 
To further verify the effects of quality control on robustness, we performed the MC simulations with strain-weighted selection scheme [7] without and with quality control of 20 spot welds for the optimized design D3. 75 samples with 100 missing spot welds have been used for both simulations. Their scatter clouds are shown in Fig. 22 along with worst case variations obtained by our proposed iterative procedure (IP) for missing of 100 most influential spot welds. Results confirm the effectiveness of quality control methodology to guarantee the impact of failure in the remaining uncontrolled spot welds within acceptable level as scatter cloud of MC simulation with QC is highly concentrated with much smaller mean values.

Thus, by controlling a reasonable number of spot welds, the robustness against missing spot welds could be guaranteed to a specific level. Nevertheless, the important question lies in finding

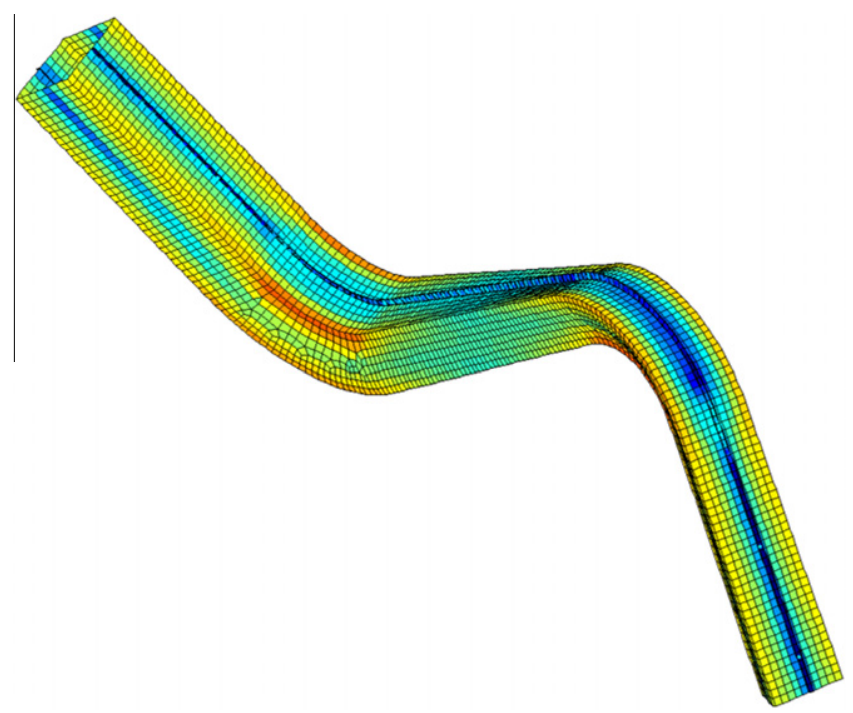

Fig. A.1. Mode 1: "bending".

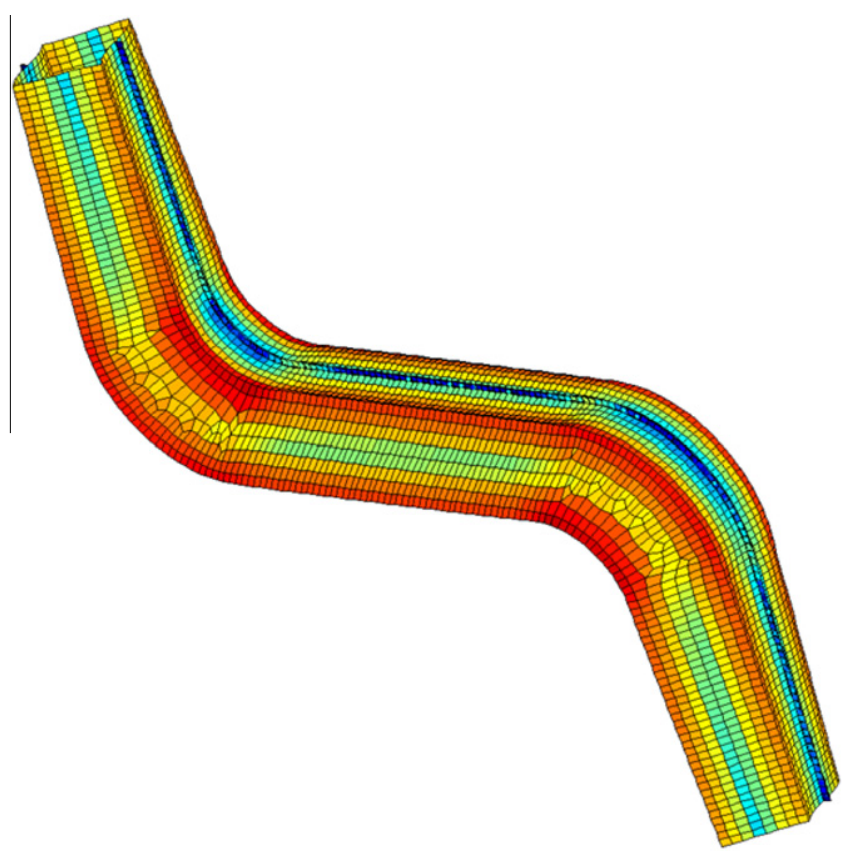

Fig. A.2. Mode 2: "torsion". an acceptable compromise between robustness and the cost of controlling additional spot welds following assembly or the cost of reinforcing critical spot welds to avoid failure during the lifetime of the vehicle.

To answer this question, the behavior of missing of the most influential uncontrolled spot welds on the eigenfrequency of mode 1 for design D2 is analyzed as a function of the number of quality controlled spot welds for maximum eigenfrequency shift of $0.5 \%$. Curves in Fig. 23 show that the design is becoming less sensitive to missing of the most influential spot welds as the number of controlled spot welds increases: with higher number of controlled spot weds, structure can sustain large uncertainty without compromising a specified performance limit.

This leads to define a trade-off curve (Fig. 24) showing the relationship between the number of RSWs structure can afford to loose against the number of quality controlled RSWs for a specific level of degradation in the performance. This curve shows that there is no gain in robustness up to a specific number of controlled spot welds but the robustness improves substantially above this number. The curves for design D3 and D4 are also plotted on the same figure for maximum eigenfrequency shift of $0.5 \%$. It illustrates that

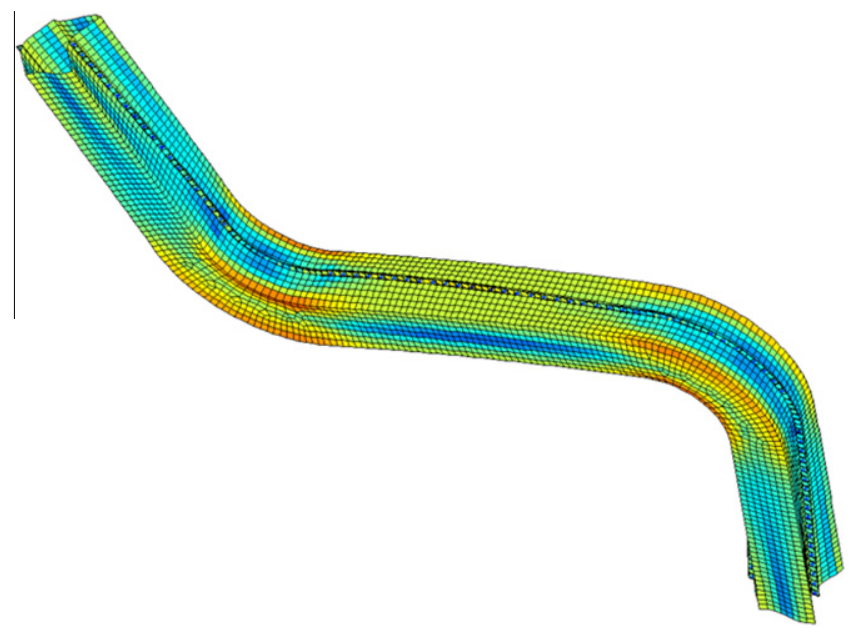

Fig. A.3. Mode 3: "bending" + deformation of section.

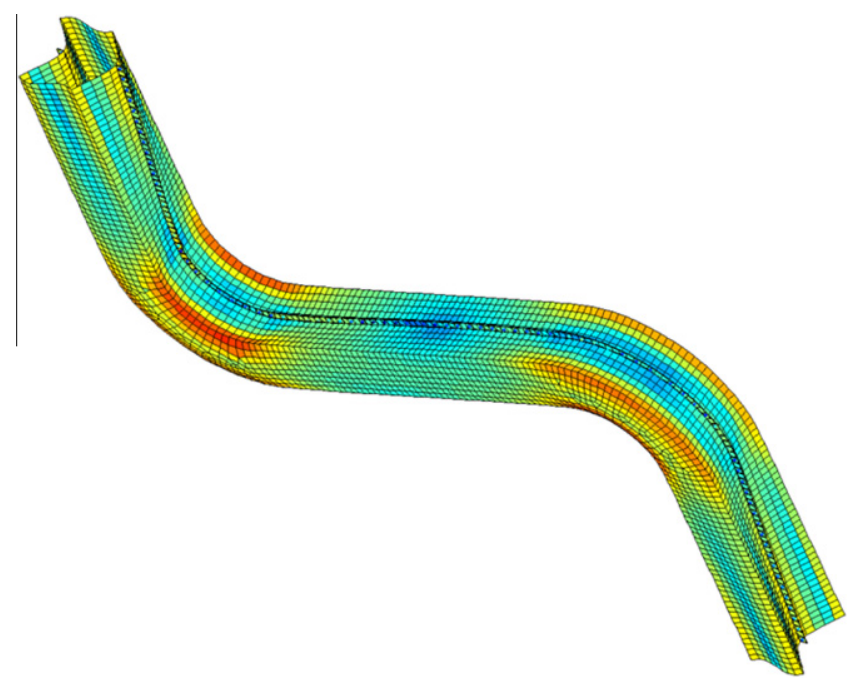

Fig. A.4. Mode 4: "bending" + deformation of section. 


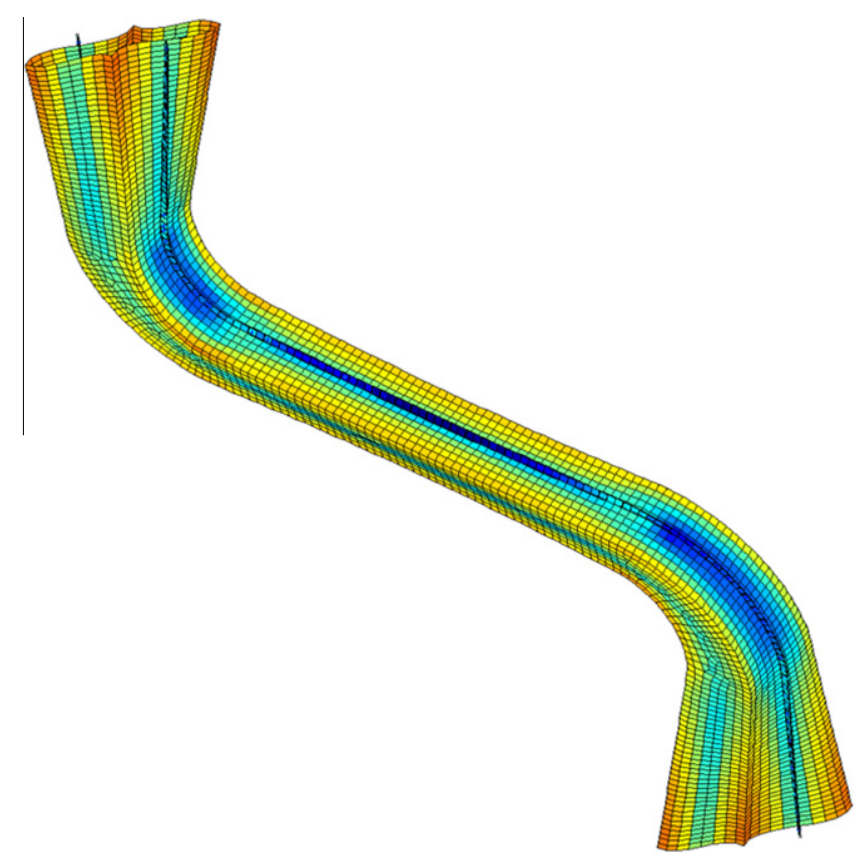

Fig. A.5. Mode 5: "bending" + deformation of section.

due to a reduction in the total number of RSWs used for assembly, the design is becoming more sensitive to the missing spot welds and more spot welds need to be quality controlled to ensure the same level of performance: to keep the variations within $0.5 \%$ where designs can afford to loose 90 spot welds, design D4 requires 50 spot welds to be quality controlled while design D3 and $\mathrm{D} 2$ require only about 25 and 15 spot welds respectively.

In short, the analyst can use this approach to select the design taking into account trade-offs between the total number of spot welds used, the impact of missing spot welds and the number of quality controlled spot welds to ensure a specific level of satisfaction within reasonable manufacturing and inspection costs.

\section{Conclusions}

An adaptive optimization procedure is presented which iteratively adds and removes spot welds to find the optimal distribution as well as the number of spot welds needed to improve the performance characteristics of interest. Meanwhile, the structural performances can be undermined by the presence of defective or missing spot welds due to manufacturing defects or fatigue. A simple approach is formulated to analyze the impact of the number of defective or missing spot welds on the system performance with the goal of replacing the more cost intensive sampling based approaches found in the literature. This approach can not only pro- vide a measure of robustness but also could serve as a useful tool to provide insight into the most influential spot welds as well as for deciding how many spot welds should be inspected following assembly. The analyst can then ensure a specific level of robustness either by quality controlling or redesigning of these small number of spot welds.

\section{Acknowledgements}

The authors thank PSA Peugeot-Citroën for providing the finite element model of the body-in-white and first author would like to thank higher education commission (HEC) of Pakistan for partial financial support.

\section{Appendix A. Mode shapes of the tube}

Figs. A.1, A.2, A.3, A.4 and A.5 show detailed views of deflection shapes of the modes of interest.

\section{References}

[1] Zhang Y, Taylor D. Optimization of spot-welded structures. Finite Elem Anal Des 2001;37(12):1013-22. doi:10.1016/S0168-874. 01)00046-4.ISSN 0168$874 X$.

[2] Chae SW, Kwon KY, Lee TS. An optimal design system for spot welding locations. Finite Elem Anal Des 2002;38(3):277-94. doi:10.1016/S0168-874. 01)00064-6.ISSN 0168-874X.

[3] Ertas AH, Sonmez FO. Optimization of spot-weld joints. Proc Inst Mech Eng Part C: J Mech Eng Sci 2009;223(3):545-55. doi:10.1243/09544062JMES1171.

[4] Wang L, Levia JP, Basu PK. Design optimization of automobile welds. Int J Veh Des 2003;31(4):377-91.

[5] Hasegawa H, Sasaki H, Uehara H, Kawamo K. Optimization of spot-weld positions for vehicle design by using hybrid meta-heuristics. Int J Veh Des 2007;43(1-4):151-72. doi:10.1504/IJVD.2007.012301.

[6] Liao YG. Optimal design of weld pattern in sheet metal assembly based on a genetic algorithm. Int J Adv Manuf Technol 2005;26(5):512-6. doi:10.1007| s00170-003-2003-5.

[7] Ouisse M, Cogan S. Robust design of spot welds in automotive structures: A decision-making methodology. Mech Syst Signal Process 2010;24(4):1172-90. doi:10.1016/i.ymssp.2009.09.012. ISSN 0888-3270.

[8] Donders S, Brughmans M, Hermans L, Liefooghe C, Van der Auweraer $\mathrm{H}$, Desmet $\mathrm{W}$. The robustness of dynamic vehicle performance to spot weld failures. Finite Elem Anal Des 2006;42(8-9):670-82. doi:10.1016/ j.finel.2005.10.012. ISSN 0168-874X.

[9] Palmonella M, Friswell MI, Mottershead JE, Lees AW. Finite element models of spot welds in structural dynamics: review and updating. Comput Struct 2005;83(8-9):648-61. doi:10.1016/j.compstruc.2004.11.003. ISSN 0045-7949.

[10] Palmonella M, Friswell MI, Mottershead JE, Lees AW. Guidelines for the implementation of the cweld and acm2 spot weld models in structural dynamics. Finite Elem Anal Des 2004;41(2):193-210. doi:10.1016/ j.finel.2004.04.003. ISSN 0168-874X.

[11] MSC.NASTRAN. MSC, 2004 edition, 2004

[12] Ouisse M, Guyader JL. Localization of structural zones producing hypersensitive behavior: finite element approach. Comput Methods Appl Mech Eng 2003;192(44-46):5001-20. doi:10.1016/S0045-782. 03)00462-6. ISSN 0045-7825.

[13] Xiang Y, Wang Q, Fan Z, Fang H. Optimal crashworthiness design of a spotwelded thin-walled hat section. Finite Elem Anal Des 2006;42(10):846-55. doi:10.1016/i.finel.2006.01.001. ISSN 0168-874X

[14] Ben-Haim y. Info-gap decision theory. 2nd ed. Oxford: Academic Press; 2006. 